\title{
Analysis of Effect of Solar Radiation Pressure of Bigger Primary on the Evolution of Periodic Orbits
}

\author{
Niraj Pathak1, V. 0. Thomas ${ }^{2}$ \\ ${ }^{1}$ Department of Mathematics, Dharmsinh Desai University, Nadiad, India \\ ${ }^{2}$ Department of Mathematics, The Maharaja Sayajirao University of Baroda, Vadodara, India \\ Email: niraj23481@yahoo.com,votmsu@gmail.com
}

How to cite this paper: Pathak, N. and Thomas, V.O. (2016) Analysis of Effect of Solar Radiation Pressure of Bigger Primary on the Evolution of Periodic Orbits. International Journal of Astronomy and Astrophysics, 6, 464-493.

http://dx.doi.org/10.4236/ijaa.2016.64037

Received: October 18, 2016

Accepted: December 24, 2016

Published: December 27, 2016

Copyright $\odot 2016$ by authors and Scientific Research Publishing Inc. This work is licensed under the Creative Commons Attribution International License (CC BY 4.0).

http://creativecommons.org/licenses/by/4.0/

\begin{abstract}
Evolution of periodic orbits in Sun-Mars and Sun-Earth systems are analyzed using Poincare surface of section technique and the effects of solar radiation pressure of bigger primary and actual oblateness of smaller primary on these orbits are considered. It is observed that solar radiation pressure of bigger primary has substantial effect on period, orbit's shape, size and their position in the phase space. Since these orbits can be used for the design of low energy transfer trajectories, so perturbations due to solar radiation pressure has to be understood and should be taken care of during trajectory design. It is also verified that stability of such orbits are negligible so they can be used as transfer orbit. For each pair of solar radiation pressure $q$ and Jacobi constant $C$ we get two separatrices where stability of island becomes zero. In this paper, detailed stability analysis of periodic orbit having two loops is given when $q=0.9845$.
\end{abstract}

\section{Keywords}

Restricted Three Body Problem, Low Energy Trajectory Design, Periodic Orbits, Solar Radiation Pressure, Poincare Surface of Section

\section{Introduction}

A Hohmann transfer (Hohmann, 1925) is effectively used to transfer a spacecraft from one circular orbit to another in a most fuel efficient way. The "low-energy" transfer trajectory [1] is used to describe the space trajectories that consume less fuel compared to Hohman transfer (Hohmann, 1925). The planar restricted three body problem (PRTBP) has long been used in celestial mechanics because it is one of the simplest non-integrable dynamic systems. RTBP refers to the motion of body of negligible mass, known as secondary body, under the sole influence of two massive primary bodies Sun-Earth, Earth- 
Moon etc. For mathematical simplicity, we shall take the total mass of primaries normalized to 1 . The mass of smaller primary is taken as $\mu$ so that the mass of its bigger counterpart is $1-\mu$. The primaries are moving in a circular orbit in counter clockwise direction about their common center of mass with angular velocity normalized to 1 . The third body, an artificial satellite or spacecraft, is moving in the same plane of motion of the primaries.

The "low-energy" transfer orbits are important in the study of practical problems regarding transfer of orbits. Low energy transfer of orbit to moon has been studied by [1]. In their study, they have considered four bodies Sun-Earth-Moon-Space craft as coupled three body systems, namely Sun-Earth-space craft and Earth-Moon-space craft. They constructed low energy transfer trajectories from the earth which executed capture at the Moon.

Study of orbits of bodies in RTBP has been described in detail by [2]. The equations of motion consist of non-linear equations and hence analytic methods seldom give physically useful results. So, a widely used method for describing the motion of secondary body (space craft) is the Poincare Surface of Section (PSS) method. [3] has given a detailed analysis of periodic orbits using PSS technique.

As per Kolmogorov-Arnold-Moser (KAM) theory (Moser, 1966), a fixed point on the Poincare surface of section represents a periodic orbit in the rotating frame, and the closed curves around the point correspond to the quasi-periodic orbits. [4] analyzed the PSS for Earth-Moon system without considering any perturbation. [5] also had analysed Sun-Mars system by incorporating perturbations due to solar radiation. They have identified periodic, quasi-periodic solutions and chaotic regions from the PSS. Similar studies have been made by [6] for Saturn Titan system. [7] analyzed the family " $P$ " orbits around smaller primary under RTBP for 14 systems under ideal conditions. Recently, [8] analyzed Sun and Saturn centered periodic orbits with solar radiation pressure and oblateness and found that there is a substantial effect of solar radiation pressure on position and geometry of infinitesimal particle orbit. [9] had studied family " $P$ " orbits and their stability for Sun-Saturn system with perturbation. In their study Sun was taken as a source of radiation and Saturn was considered as oblate spheroid. By taking the actual coefficient of oblateness for Saturn and different values of solar radiation pressure, the family " $p$ " orbits has been analyzed in detail.

Periodic orbits of spacecraft around the two massive primaries are used to construct low energy trajectory. In this paper, we have studied periodic orbits around both primaries in the frame work of RTBP using PSS method. [10] established three classes with orbits around both primaries depending on motion of spacecraft is prograde or retrograde in the rotating system as well as fixed system. In this paper we have analyzed periodic orbits around both primaries with retrograde motion in rotating system and analyzed periodic orbits having number of loops from 1 to 5 for different pairs of solar radiation pressure $q$ and Jacobi constant $C$ for Sun-Mars and Sun-Earth system. It has been found that $q$ and $C$ have substantial effect on the position in phase space, shape and size of the orbits and hence must be considered during low energy trajectory design. 


\section{Equations of Motion}

Let $m_{1}$ and $m_{2}$ be two masses of first and second primary bodies, respectively. Mass of first primary is greater than mass of second primary. The solar radiation pressure force $F_{p}$ changes with the distance by the same law as the gravitational attraction force $F_{g}$ and acts opposite to it. It is possible to consider that the result of the action of this force leads to reducing the effective mass of the sun [5]. Accordingly, the sun's resultant force acting on the particle is

$$
F=F_{g}-F_{p}=F_{g}\left(1-\frac{F_{p}}{F_{g}}\right)=q F_{g},
$$

where $q=1-\frac{F_{p}}{F_{g}}$, known as the mass reduction factor constant for the given particle.

We follow the notation and terminology of [2]. It is clear from Equation (1) that $q=1$ indicates $F=F_{g}$ and hence the solar radiation pressure drops to zero.

The perturbed mean motion $n$ of second primary body is given by,

$$
n^{2}=1+\frac{3}{2} A_{2}
$$

where

$$
A_{2}=\frac{\rho_{e}^{2}-\rho_{p}^{2}}{5 R^{2}} .
$$

Here $A_{2}$ is oblateness coefficient of second primary. $\rho_{e}$ and $\rho_{p}$ represent equatorial and polar radii of second primary and $R$ is the distance between two primaries.

The unit of mass is chosen equal to the sum of the primary masses and the unit of length is equal to their separation. The unit of time is such that the Gaussian constant of gravitation is unity in the unperturbed case. The usual dimensionless synodic coordinate system $O x y$ is used to express the motion of the secondary body (space craft).

Choose a rotating coordinate system with origin at the center of mass, the primaries lie on the $x$-axis at the points $(-\mu, 0)$ and $(1-\mu, 0)$, respectively, where mass factor $\mu=\frac{m_{2}}{\left(m_{1}+m_{2}\right)}$. Following [11], the equations of motion of the spacecraft are

$$
\ddot{x}-2 n \dot{y}=\frac{\partial \Omega}{\partial x}, \quad \ddot{y}+2 n \dot{x}=\frac{\partial \Omega}{\partial y},
$$

where

$$
\Omega=\frac{n^{2}}{2}\left[(1-\mu) r_{1}^{2}+\mu r_{2}^{2}\right]+\frac{q(1-\mu)}{r_{1}}+\frac{\mu}{r_{2}}+\frac{\mu A_{2}}{2 r_{2}^{3}} .
$$

Here

$$
r_{1}^{2}=(x-\mu)^{2}+y^{2}
$$

and

$$
r_{2}^{2}=(x+1-\mu)^{2}+y^{2}
$$


System of Equation (4) lead to the first integral

$$
\dot{x}^{2}+\dot{y}^{2}=2 \Omega-C
$$

where $C$ is Jacobi constant of integration given by

$$
C=n^{2}\left[(1-\mu) r_{1}^{2}+\mu r_{2}^{2}\right]+2 \frac{q(1-\mu)}{r_{1}}+2 \frac{\mu}{r_{2}}+\frac{\mu A_{2}}{r_{2}^{3}}-\dot{y}^{2} .
$$

These equations of motion are integrated in $(x, y)$ variables using a Runge-Kutta Gill fourth order variable or fixed step-size integrator. The initial conditions are selected along the $x$-axis. By defining a plane, say $y=0$, in the resulting three dimensional space the values of $x$ and $\dot{x}$ can be plotted every time the particle has $y=0$, whenever the trajectory intersects the plane in a particular direction, say $\dot{y}>0$. We have constructed Poincare surface section (PSS) on the $x, \dot{x}$ plane. The initial values were selected along the $O x$-axis by using intervals of length 0.001 . By giving different value of $C$ we can plot the trajectories, and then analysis of orbits can be done.

\section{Results and Discussion}

We shall consider two systems, the Sun-Mars system and the Sun-Earth system. The masses of Sun, Earth and Mars are taken as $1.9881 \times 10^{30} \mathrm{~kg}, 5.972 \times 10^{24} \mathrm{~kg}$, and $6.4185 \times$ $10^{23} \mathrm{~kg}$, respectively [5]. The mass factor $\mu$, for the Sun-Earth and Sun-Mars systems, are 0.000003002 and 0.0000003212 , respectively. Equatorial and polar radii of Earth are $6378.1 \mathrm{kms}$. and $6356.8 \mathrm{kms}$. and that of Mars are $3396.2 \mathrm{kms}$. and $3376.2 \mathrm{kms}$., respectively. The distance between Sun and Earth is taken as 149,600,000 kms. and distance between Sun and Mars is 227,940,000 kms. So, oblateness coefficient calculated from Equation (2) for Sun-Earth and Sun-Mars have values $A_{2}=2.42405 \times 10^{-12}$ and $A_{2}=5.21389 \times 10^{-13}$ respectively. $q=1$ means that there is no perturbation due to solar radiation pressure and $q<1$ indicates that we are including perturbation due to solar radiation pressure. For a given $q$, selection of $C$ is not arbitrary. By solving Equation (9) w.r.t. $\dot{x}^{2}$ and by setting in to this equation $y=0$ and $\dot{y}^{2}>0$ the resulting inequality defines the regions allowed to motion on the PSSs. Thus, we eliminate the possibility of excluded region for secondary body between Sun and Earth (or Mars). This maximum value of $C$ is considered as admissible value of $C$.

Table 1 and Table 2 shows range of admissible values of $C$ for Sun-Earth and SunMars system. It can be observed that for Sun-Mars system size of excluded region is more than size of excluded region of Sun-Earth system. So, we can say that as mass factor increases, size of excluded region decreases. We have analyzed the effect of solar radiation pressure on admissible value of Jacobi constant $C$. We have studied the effect of solar radiation pressure on the location and period of Sun-Mars system for different values of Jacobi constant $C$ using PSS. Figure 1 displays the PSS for Sun-Mars system for the pair $(q, C)$ as $(0.9845,2.94)$ by taking value of $x$ from the interval $[0.8,1]$ with interval of $x$ differencing for $x$ as 0.001 . Also, time span $t=10,000$ time units and interval of differencing for time is taken as 0.001 . So, for each $x$ equations of motions are 
Table 1. Admissible range of $C$ for Sun-Earth system.

\begin{tabular}{ccccc}
\hline$q$ & Maximum value of $C$ & $\begin{array}{c}\text { Value of } C \text { greater than } \\
\text { maximum value }\end{array}$ & $\begin{array}{c}\text { Excluded region (where } y \\
\text { is complex) }\end{array}$ & Size of excluded region \\
\hline 1 & 3.0 & 3.001 & $x=0.988$ to $x=0.994$ & 0.006 \\
0.995 & 2.99 & 2.991 & $\mathrm{x}=0.985$ to $\mathrm{x}=0.994$ & 0.009 \\
0.99 & 2.98 & 2.981 & $\mathrm{x}=0.983$ to $\mathrm{x}=0.995$ & 0.012 \\
0.9845 & 2.969 & 2.970 & $\mathrm{x}=0.98$ to $\mathrm{x}=0.995$ & 0.015 \\
\hline
\end{tabular}

Table 2. Admissible range of $C$ for Sun-Mars system.

\begin{tabular}{ccccc}
\hline$q$ & Maximum value of $C$ & $\begin{array}{c}\text { Value of } C \text { greater than } \\
\text { maximum value }\end{array}$ & $\begin{array}{c}\text { Excluded region (where } y \\
\text { is complex) }\end{array}$ & Size of excluded region \\
\hline 1 & 3.0 & 3.001 & $x=0.984$ to $x=1.0$ & 0.016 \\
0.995 & 2.99 & 2.991 & $\mathrm{x}=0.982$ to $\mathrm{x}=1.0$ & 0.018 \\
0.99 & 2.98 & 2.981 & $\mathrm{x}=0.980$ to $\mathrm{x}=1.0$ & 0.020 \\
0.9845 & 2.969 & 2.970 & $\mathrm{x}=0.978$ to $\mathrm{x}=0.995$ & 0.022 \\
\hline
\end{tabular}

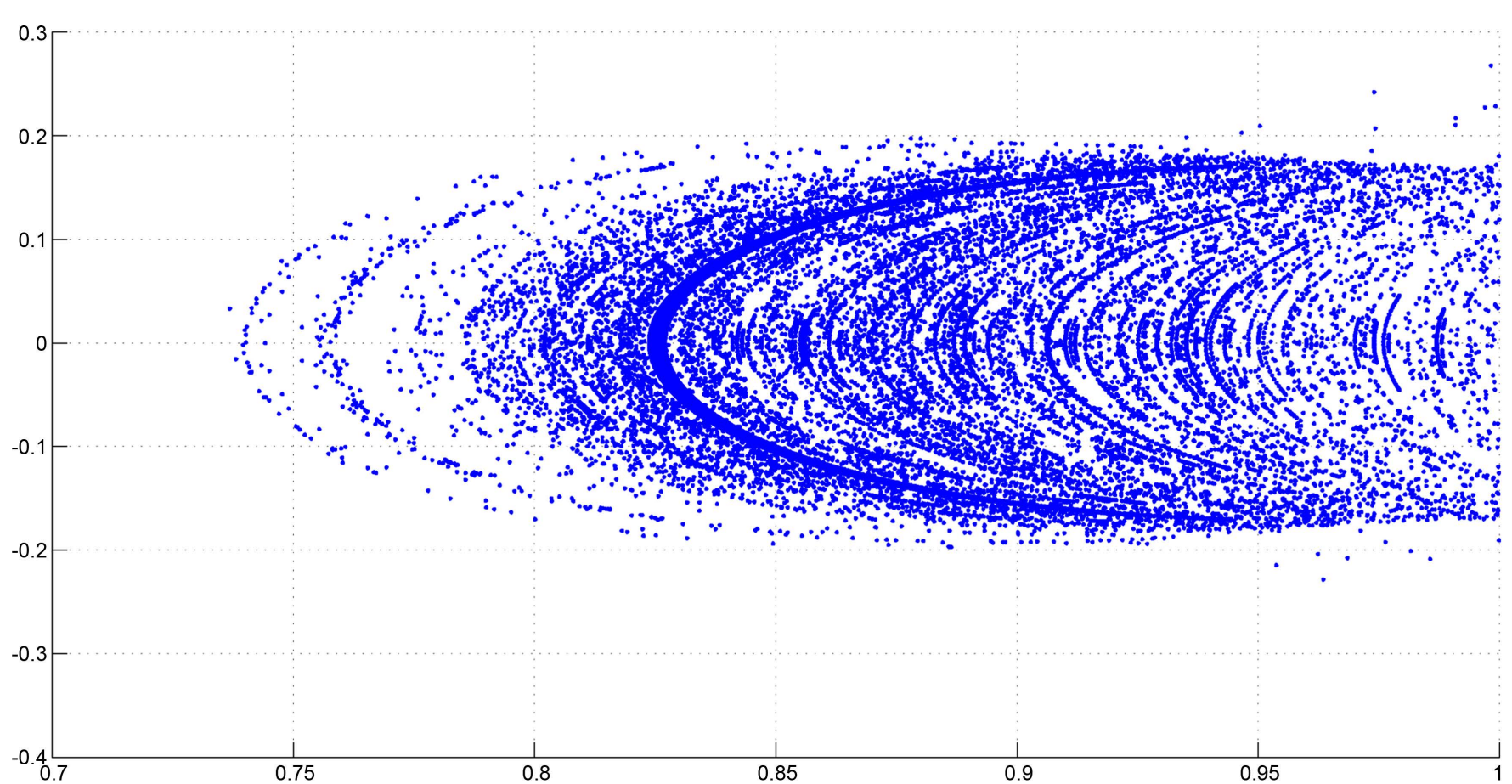

Figure 1. PSS for $q=0.9845$ and $C=2.94$, for $x=[0.8,1], t=10,000$ for Sun-Mars system.

integrated using Runge-Kutta-Gill method. Each solution is plotted as a point in the $x-\dot{x}$-plane in Figure 1. The arcs of PSS are known as islands whose center gives periodic orbits.

In a similar way, we have obtained PSS for Sun-Earth system as shown in Figure 2. This PSS is also constructed for the pair $(q, C)$ given by $(0.9845,2.94)$. Our aim is to make a comparative study of the effect of solar radiation pressure on different parameters of the orbits of the secondary body (space craft) in the Sun-Earth and Sun-Mars systems using PSS technique. Mass factor $\mu$ of Sun-Earth is greater than Sun-Mars. 
The numerical values of location and period of periodic orbit of spacecraft for $C=$ 2.93, 2.94, 2.95, 2.96 and for $q=1,0.995,0.99$ and 0.9845 are displayed in Table 3. It is

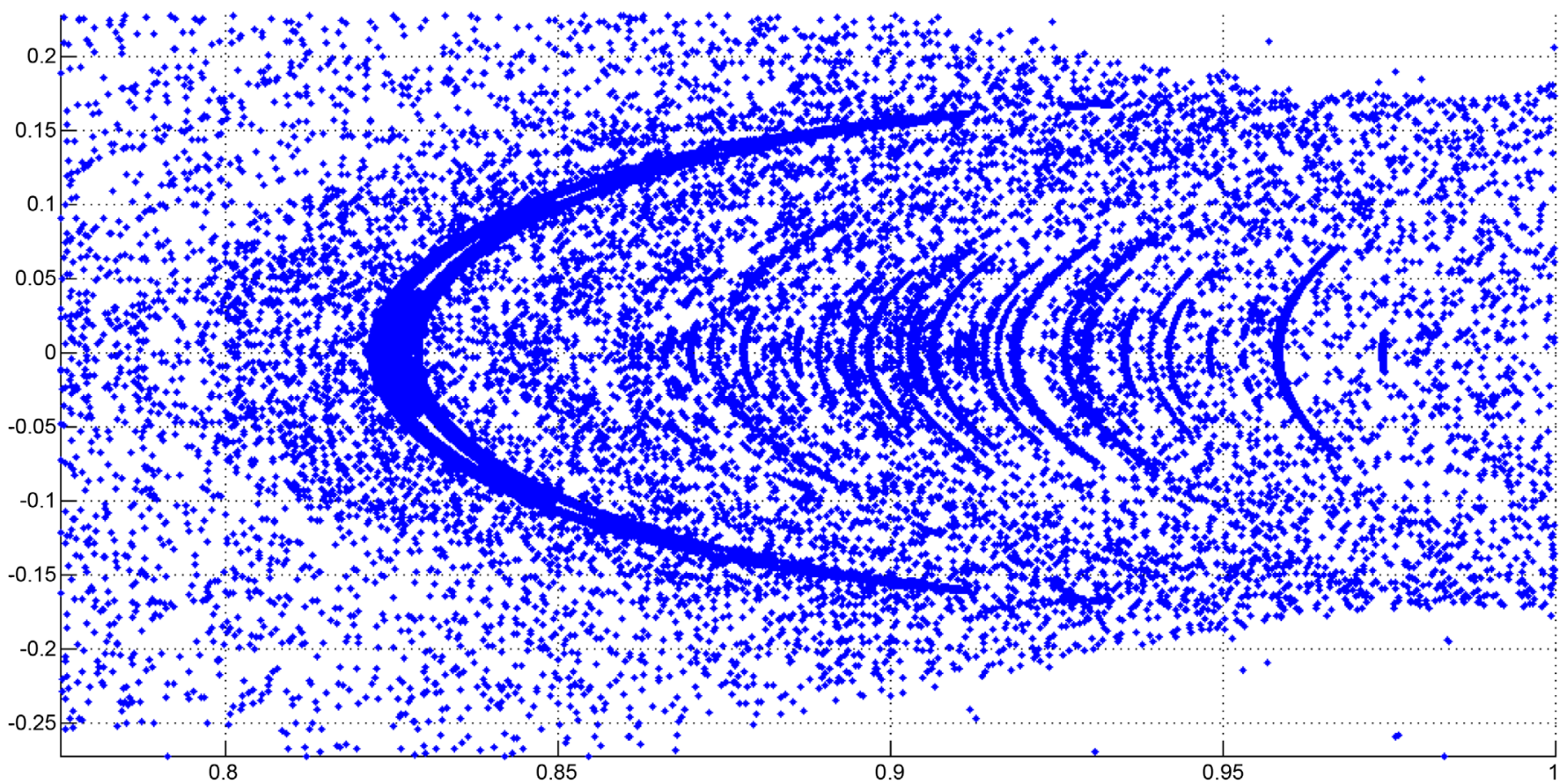

Figure 2. PSS for $q=0.9845$ and $C=2.94$, for $x=[0.8,1], t=10,000$ for Sun-Earth system.

Table 3. Analysis of periodic orbit for different pairs of $q$ and $C$ for Sun-Mars system.

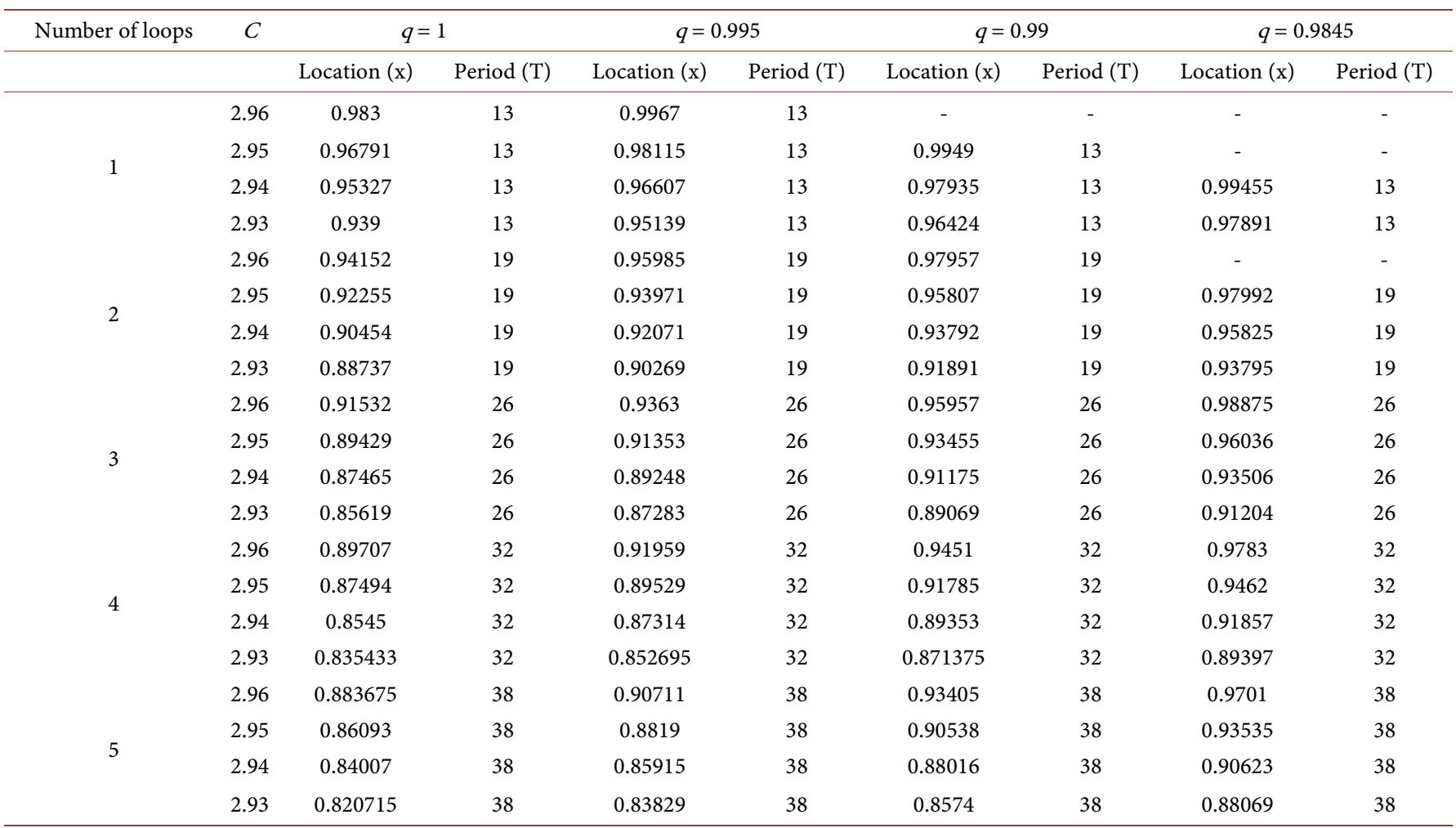


observed from the table that a change in $C$ in the range $(2.93,2.96)$ affects the location but has no effect on the period and number of loops of the orbit. Solar radiation pressure also affects the location and period of the orbit. Similarly, the effects of $C$ and $q$ in the location and period for the Sun-Earth system are studied and the numerical estimates of the changes are displayed in Table 4. It can be seen that, for both the systems, the period of orbit increases with increase in the number of loops.

A noticeable difference observed in both the systems is that for $C=2.96, q=0.995$, single-loop periodic orbit, for $C=2.96, q=0.99$ two loops orbit, for $C=2.96, q=$ 0.9845 , three loops periodic orbit does not exist for Sun-Earth system where as it exists for Sun-Mars system.

The periodic orbits starting from single-loop to five loops in the Sun-Mars system for $q=0.9845$ and 0.995 are shown in Figures 3(a)-(j). It can be observed, from Figure 3 and Figure 4, that the width of the orbit decreases continuously as the number of loops increases. Also, as perturbation due to solar radiation pressure decreases from $q=$ 0.9845 to 0.995 we can observe that size of the loop increases. Further, it can be noticed that as the perturbation due to solar radiation pressure increases (that is, $q$ decreases), the location of the closest approach of the space craft shifts towards the second primary body in both Sun-Mars and Sun-Earth system. This is contrary to the effect of oblateness of the second primary. That is, as the oblateness coefficient increases the position of the

Table 4. Analysis of periodic orbit for different pairs of $q$ and $C$ for Sun-Earth system.

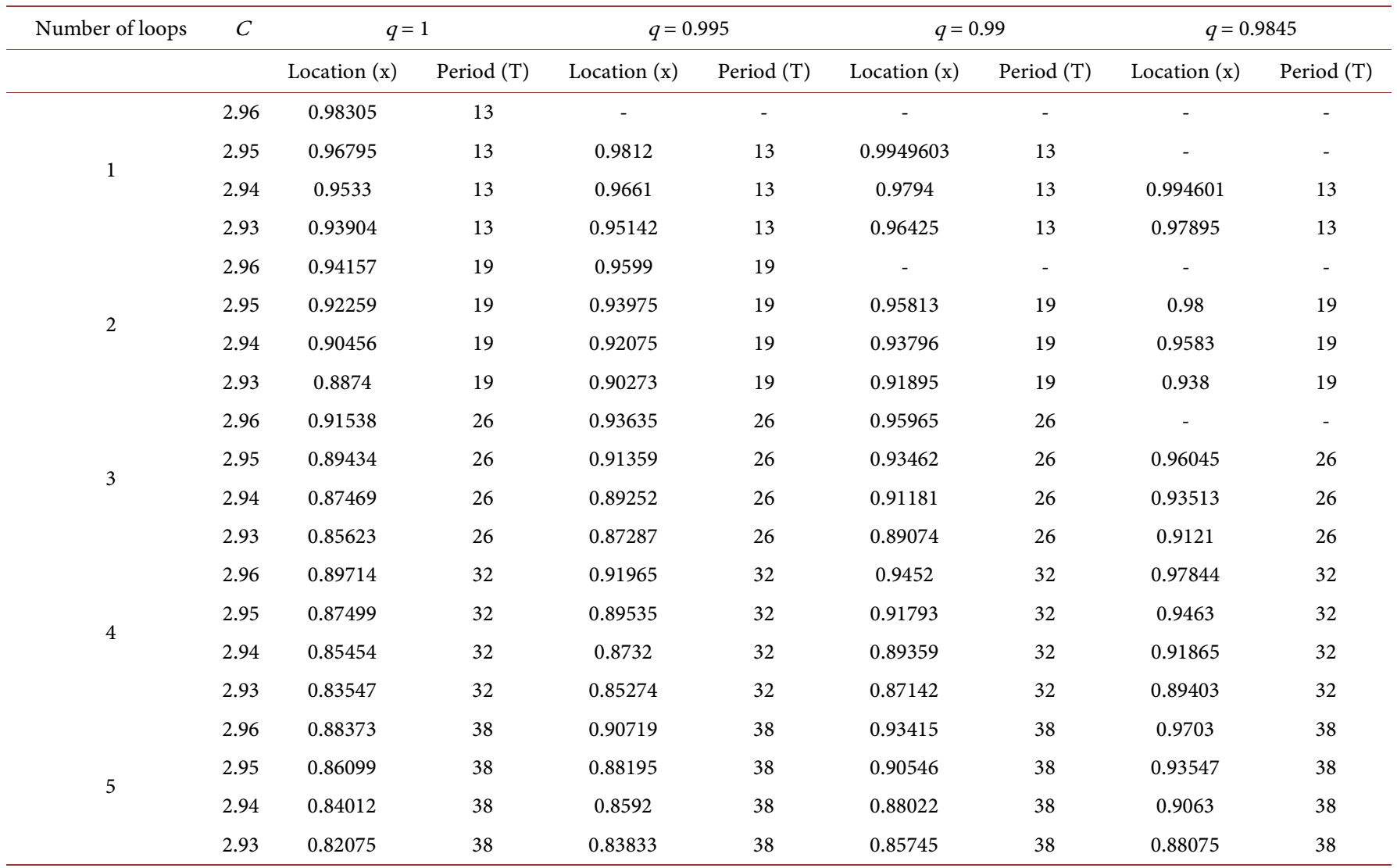




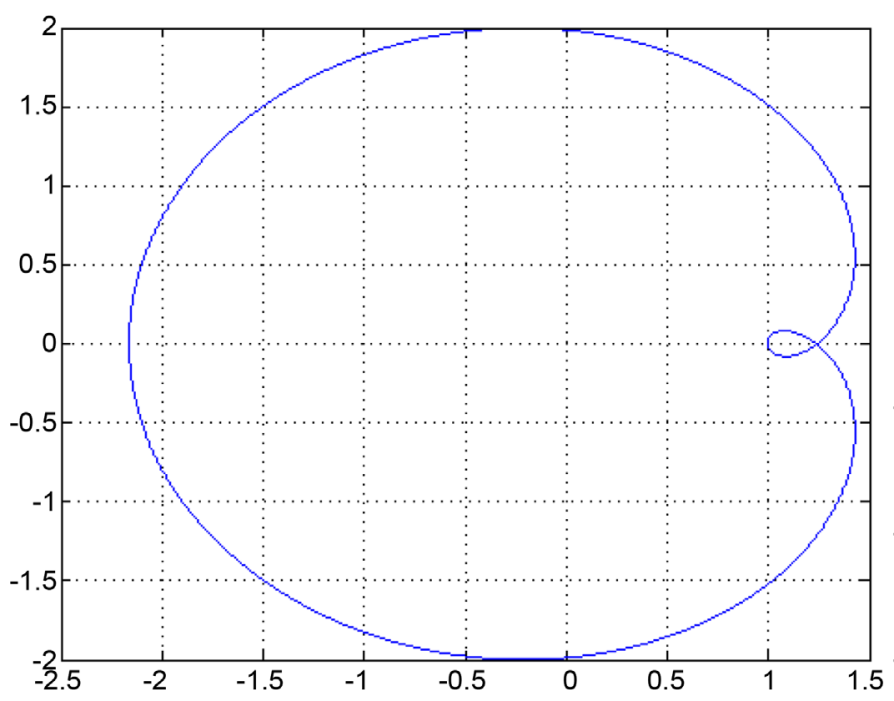

(a) $q=0.9845$, single loop periodic orbit.

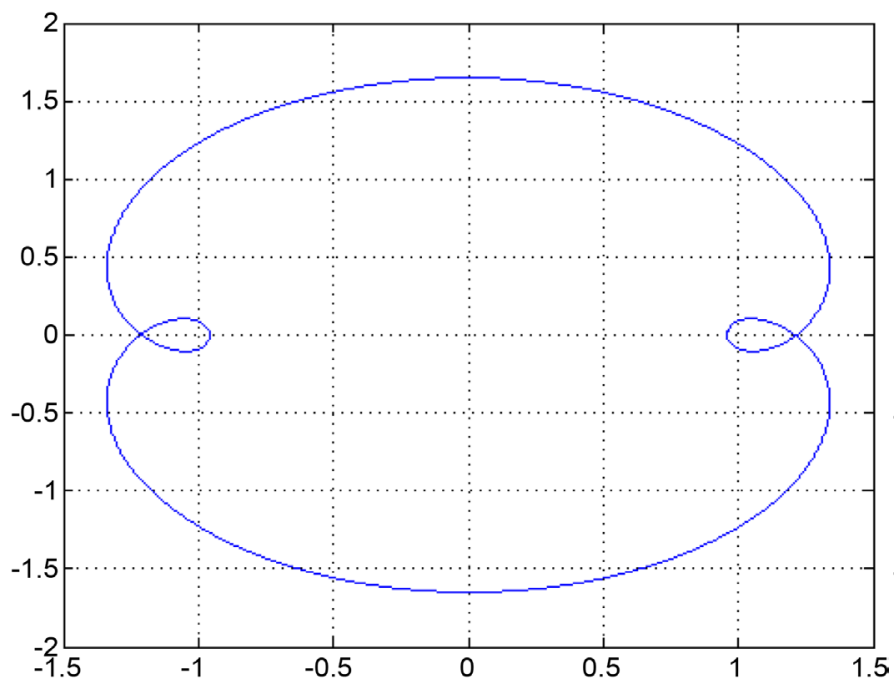

(c) $q=0.9845$, two loops periodic orbit.

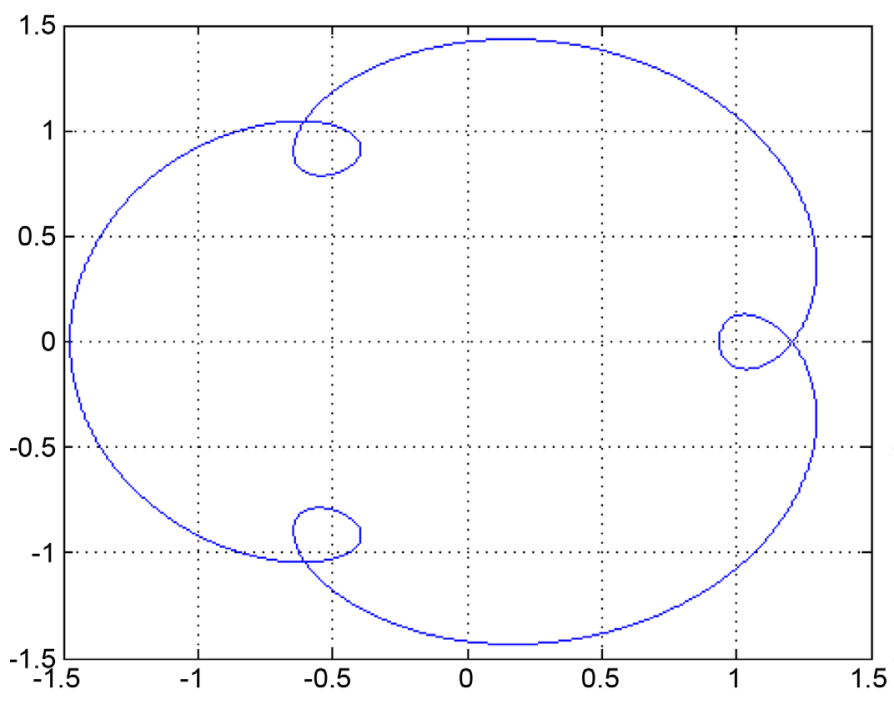

(e) $q=0.9845$, three loops periodic orbit.

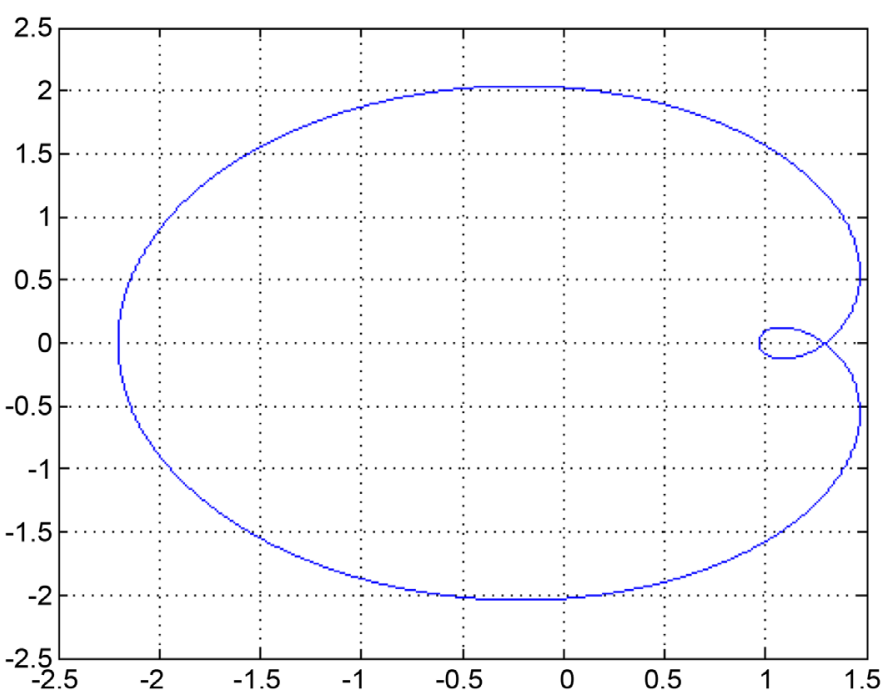

(b) $q=0.995$, single loop periodic orbit.

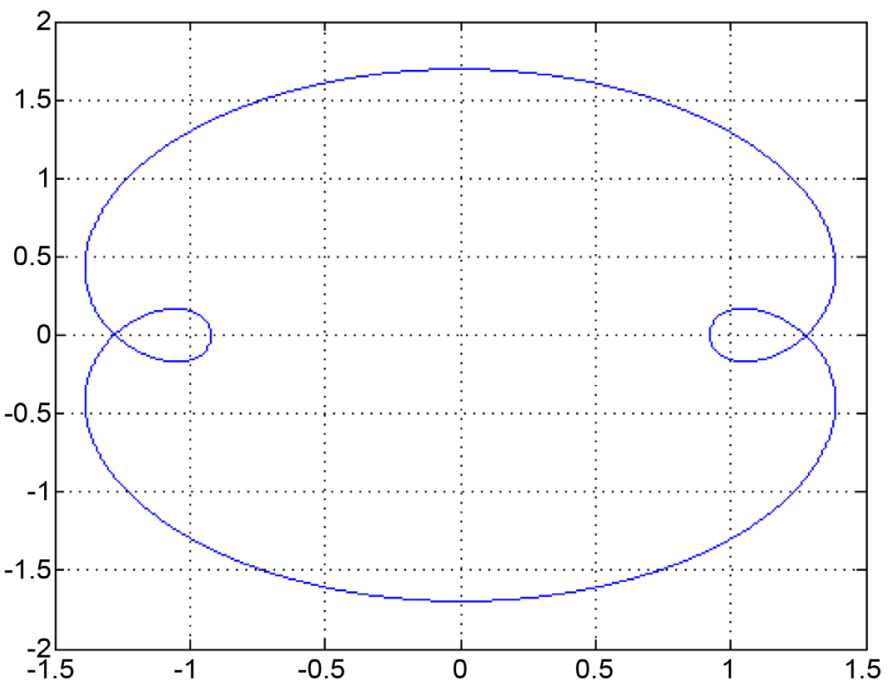

(d) $q=0.995$, two loops periodic orbit.

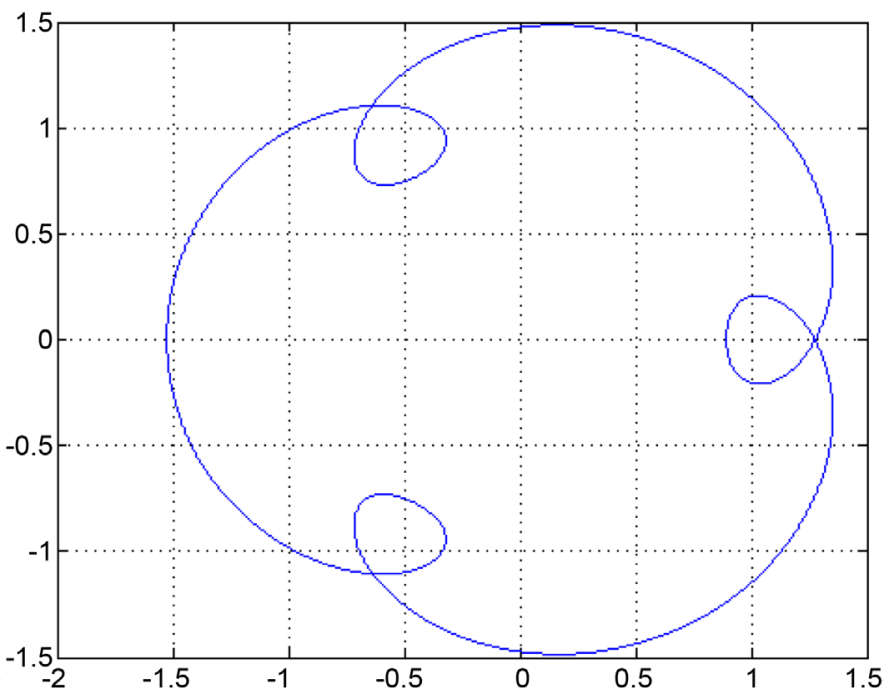

(f) $q=0.995$, three loops periodic orbit. 


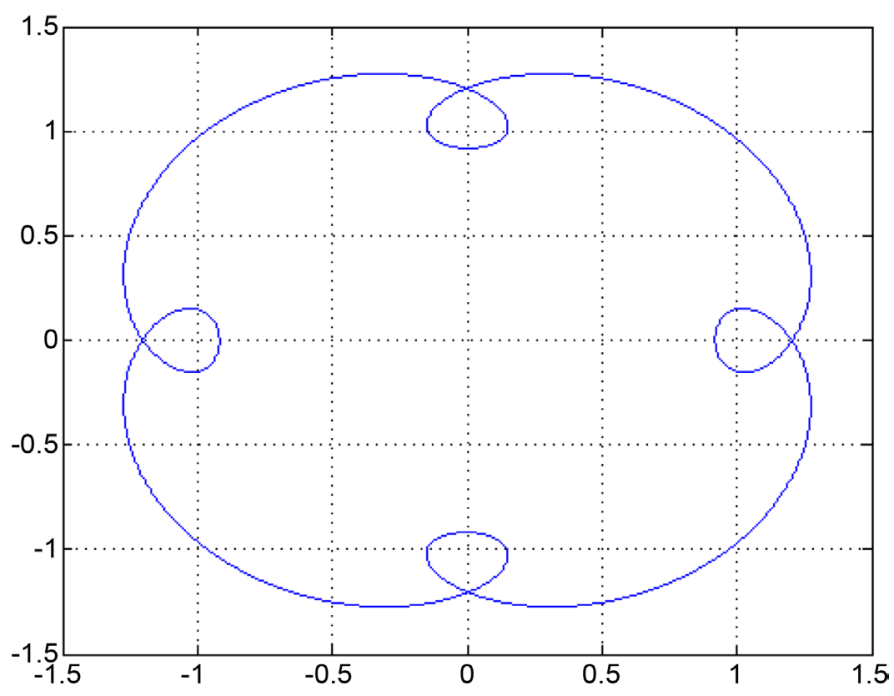

(g) $q=0.9845$, four loops periodic orbit.

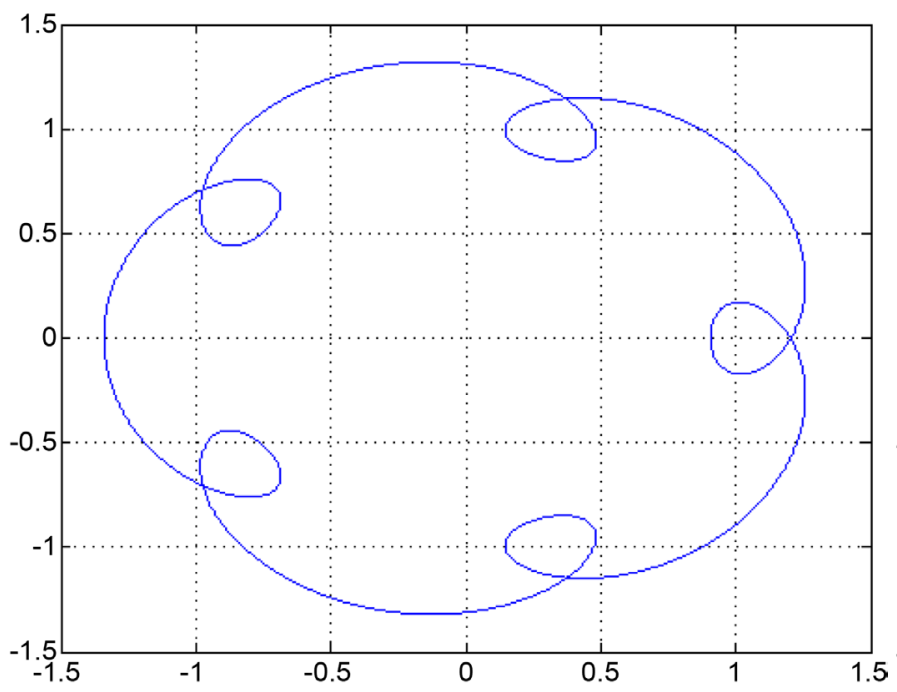

(i) $q=0.9845$, five loops periodic orbit.

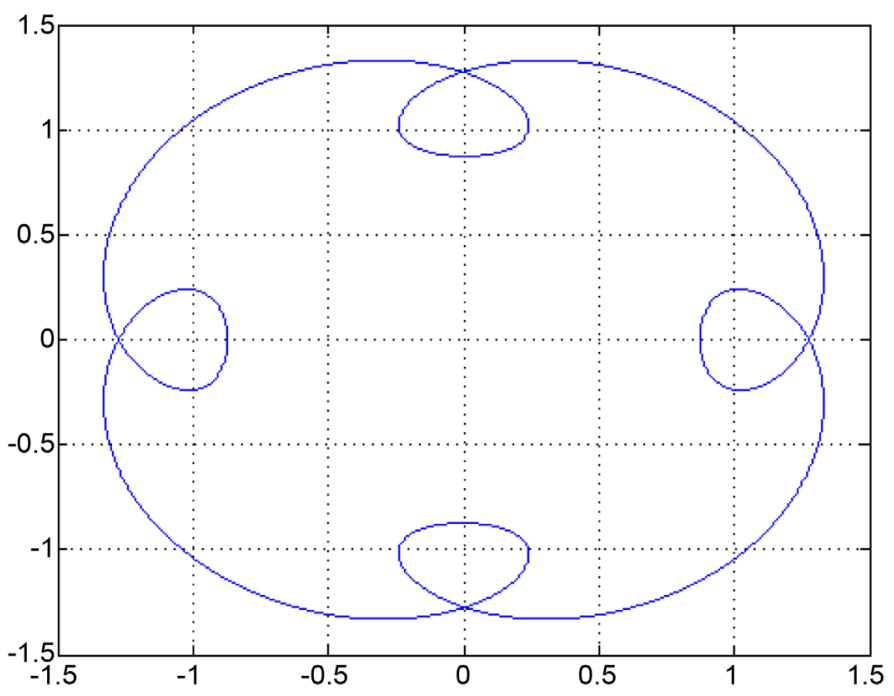

(h) $q=0.995$, four loops periodic orbit.

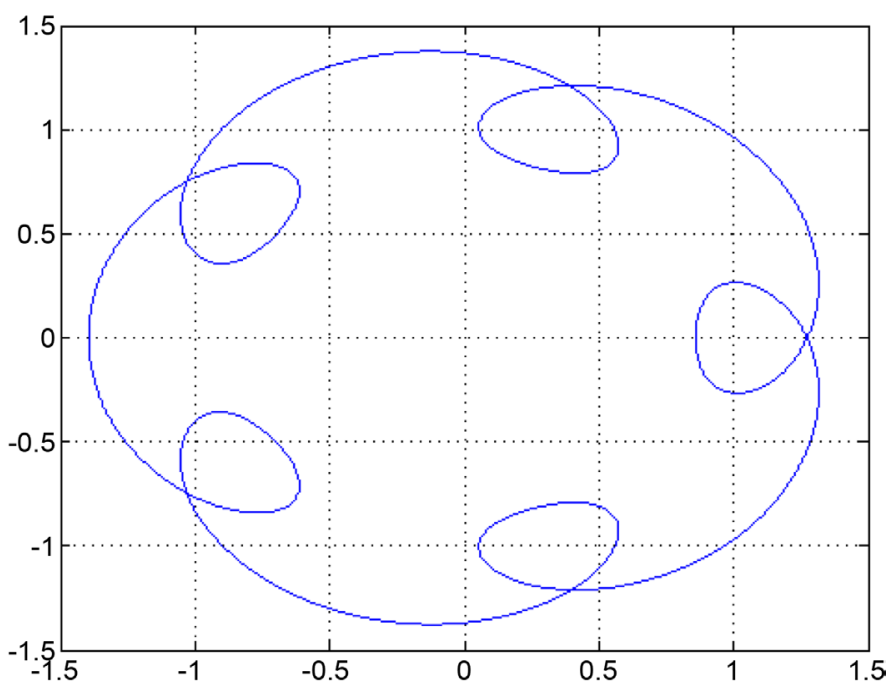

(j) $q=0.995$, five loops periodic orbit.

Figure 3. Periodic orbits around both primaries for Sun-Mars system for $C=2.94$.

closest approach of the space craft receeds from the second primary [9]. Further in all cases the infinitesimal particle (space craft) orbits around the second primary (Mars) in addition to orbiting both primaries. Further the secondary body is closest to Mars in the single loop closed orbit. Such orbits may be useful in the study of different aspects of both primaries. In many models available in literature not many closed orbits possess this kind of nature. We can observe similar nature in the orbits shown in Figures $4(a)-(j)$ in the case of Sun-Earth system.

Figure 3 and Figure 4 depict periodic orbits with number of loops varying from 1 to 5 with different solar radiation pressure for Sun-Mars and Sun-Earth systems, respectively. For both of these figures value of Jacobi constant $C$ is 2.94 . The expression for semi-major axis $a$ and eccentricity $e$ of the periodic orbits around both primaries are given by [3]. 


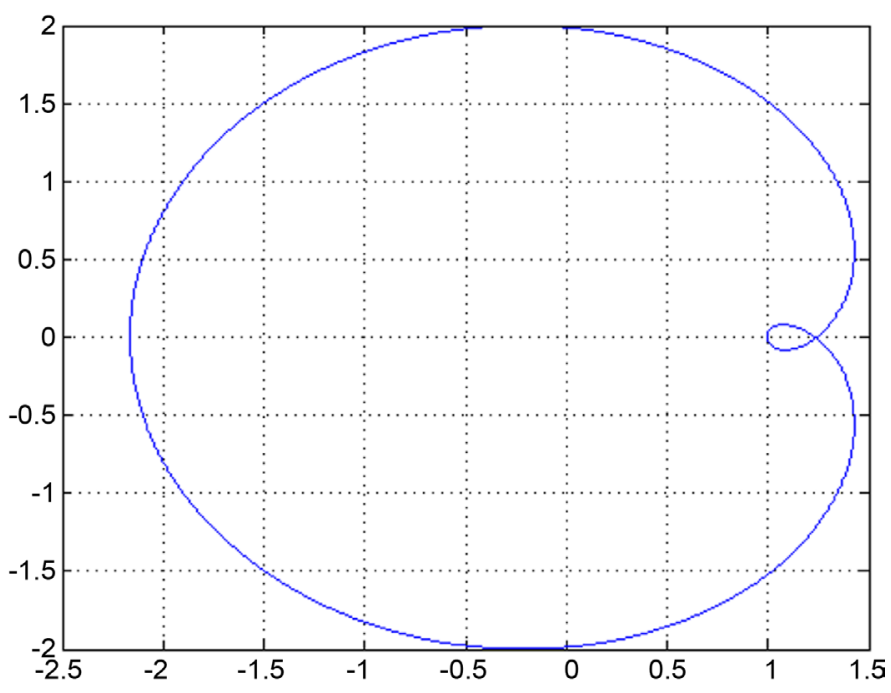

(a) $q=0.9845$, single loop periodic orbit.

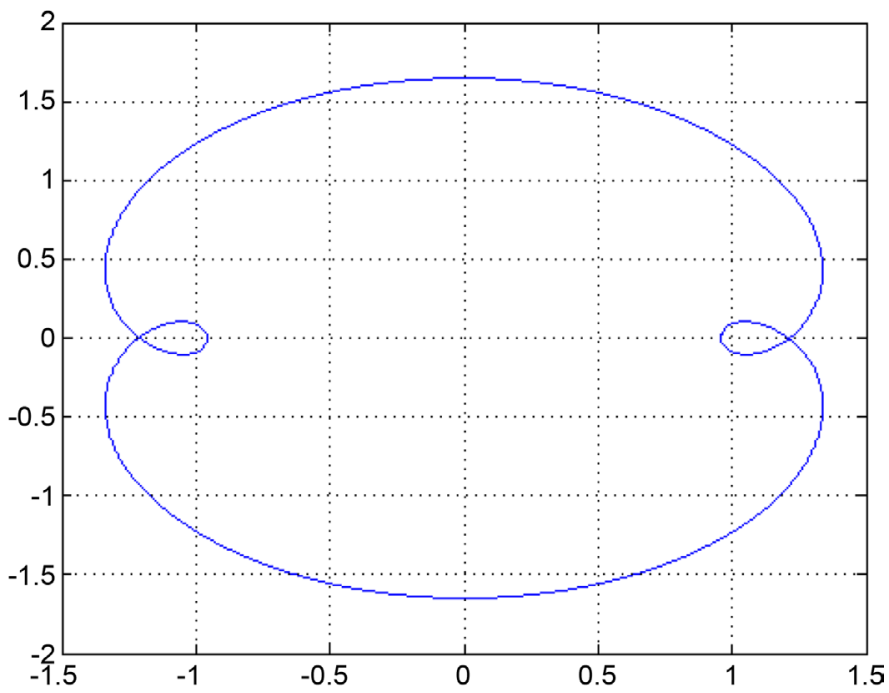

(c) $q=0.9845$, two loops periodic orbit.

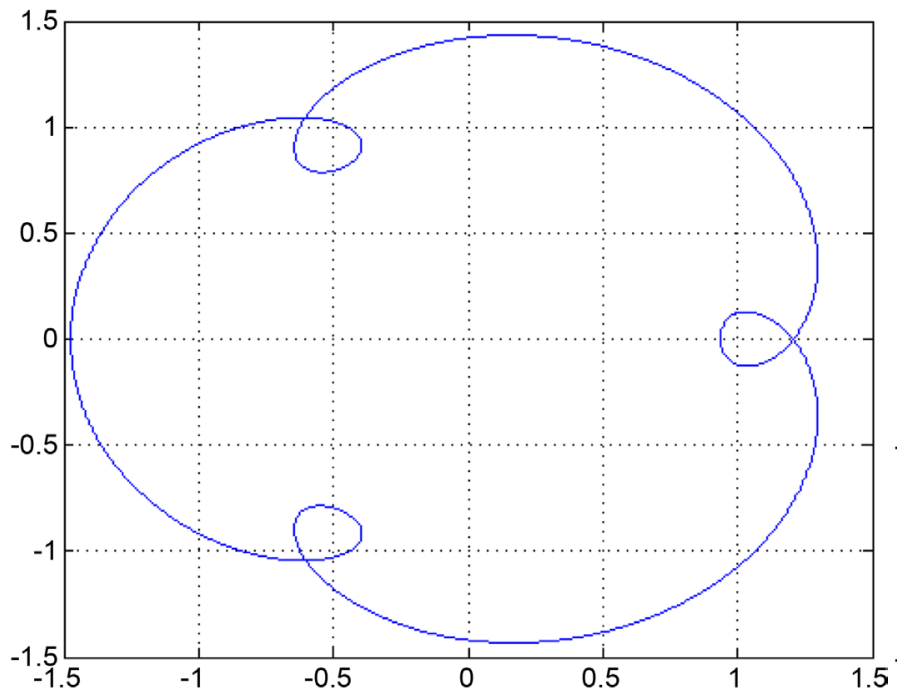

(e) $q=0.9845$, three loops periodic orbit.

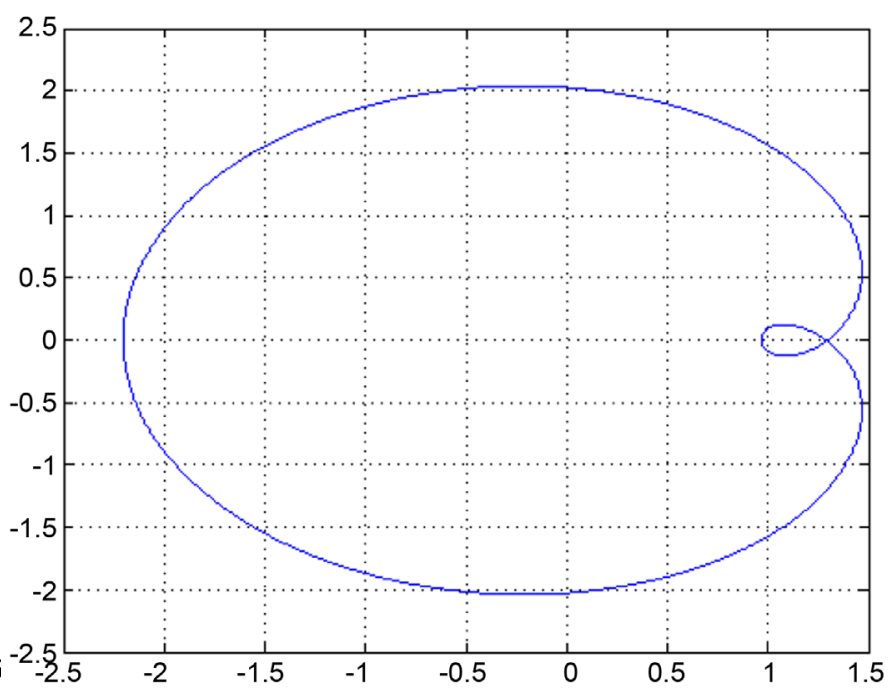

(b) $q=0.995$, single loop periodic orbit.

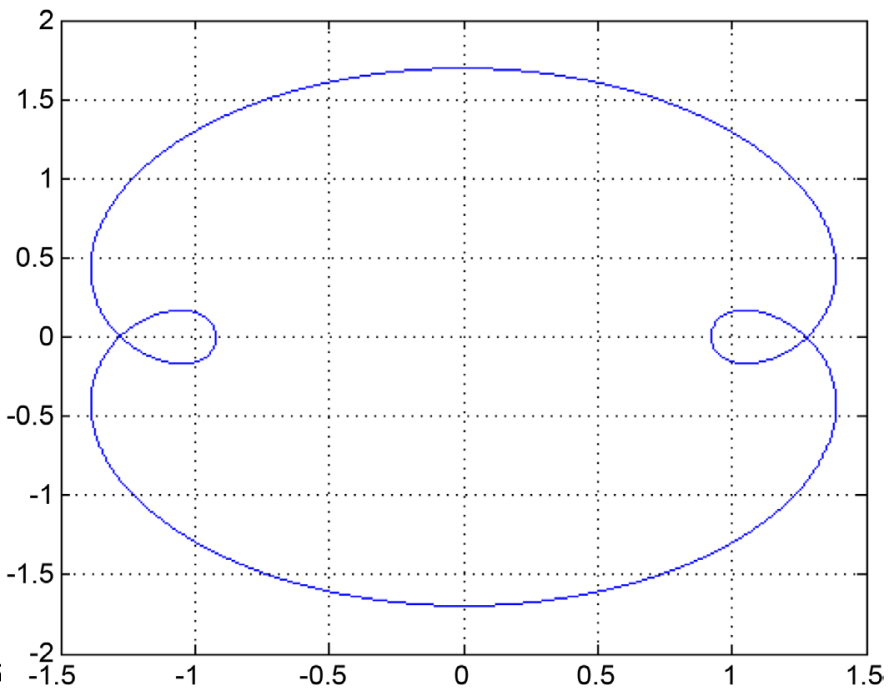

(d) $q=0.995$, two loops periodic orbit.

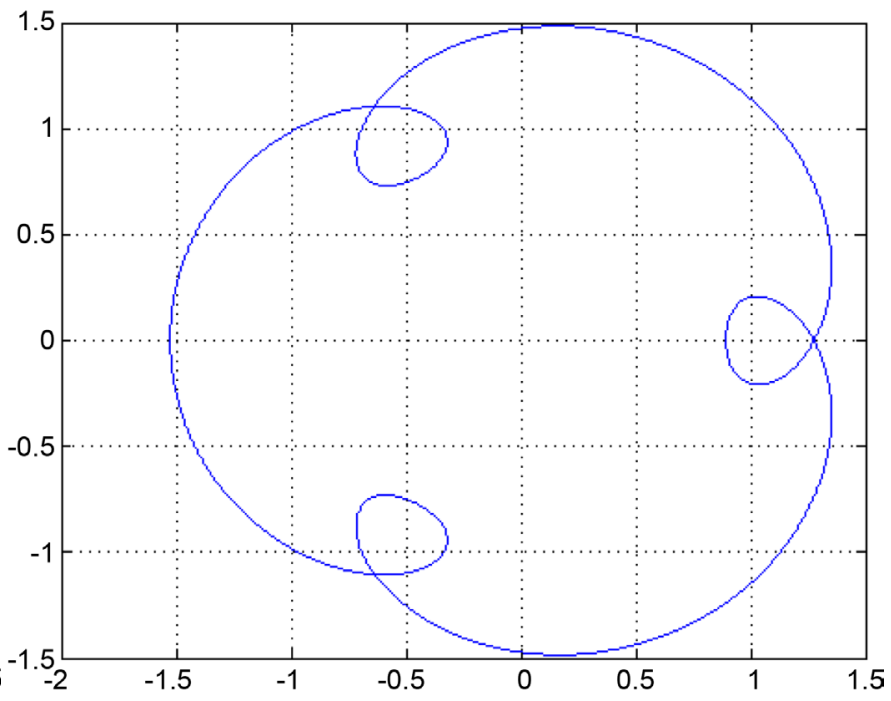

(f) $q=0.995$, three loops periodic orbit. 

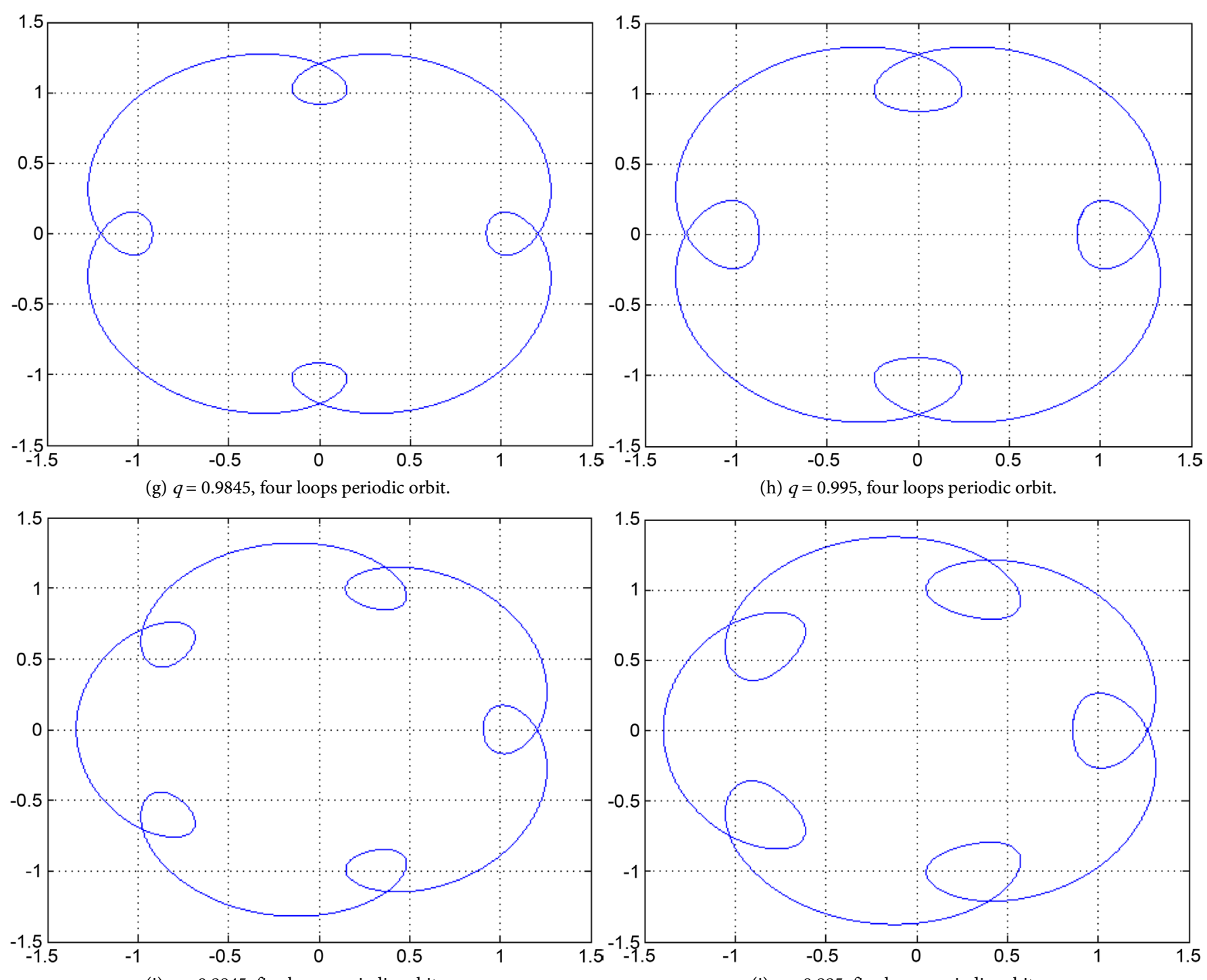

(i) $q=0.9845$, five loops periodic orbit.

(j) $q=0.995$, five loops periodic orbit.

Figure 4. Periodic orbits around both primaries for Sun-Earth system for $C=2.94$.

$$
\begin{aligned}
& a=\left[\frac{2}{r_{1}}-\frac{V^{2}}{1-\mu}\right]^{-1}, \\
& e=\sqrt{1-\frac{h^{2}}{a(1-\mu)}},
\end{aligned}
$$

where,

$$
\begin{gathered}
V^{2}=\left(\dot{x}^{2}+(\dot{y}+x+\mu)^{2}\right), \\
h=\left(x+\mu_{2}\right)(y+\dot{x}+\mu), \\
r_{1}^{2}=(x+\mu)^{2}+y^{2} .
\end{gathered}
$$

Using the set of Equations (10)-(14), for different number of loops and selected val- 
ues of Jacobi constant $C$ and solar radiation pressure $q$, the location of the orbit, the semi major axis $a$ and eccentricity $e$ of the periodic orbits are calculated and numerical values are given for Sun-Mars system in Table 5.

From Table 5, it can be noticed that for a fixed number of loops, a decrease in the value of Jacobi constants from 2.96 to 2.93 cause the orbits to recede from the Mars; the semi-major axis decreases and the eccentricity of the orbit increases. Similar effects can be observed for Sun-Earth system and the numerical estimates of these effects are displayed in Table 6.

We have studied the variation of position of periodic orbits around Sun-Mars and Sun-Earth system due to the variation in solar radiation pressure and Jacobi constants $C$. In Figure 5 we have shown the variation of position of closed periodic orbit with one loop for solar radiation pressure in the range $(0.9845,1)$ for Sun-Mars system corresponding to Jacobi constants $C=2.93,2.94$. From Figure 5 it is clear that the position of the orbits recedes from Mars when the solar radiation pressure decreases from 0.9845 to 1 and $C$ decreases. Note that Single-loop periodic orbits are not available for $C=2.95$ and 2.96 .

Similar kind of conclusion can be drawn from Figure 6 for the Sun-Earth system.

We have studied the effect of $q$ and $C$ on the position of the orbits having loops varying from 1 to 5 for both Sun-Mars and Sun-Earth systems. The results of these

Table 5. Location, semi major axis and eccentricity of orbits for $q=1,0.995,0.99$ and 0.9845 for Sun-Mars system.

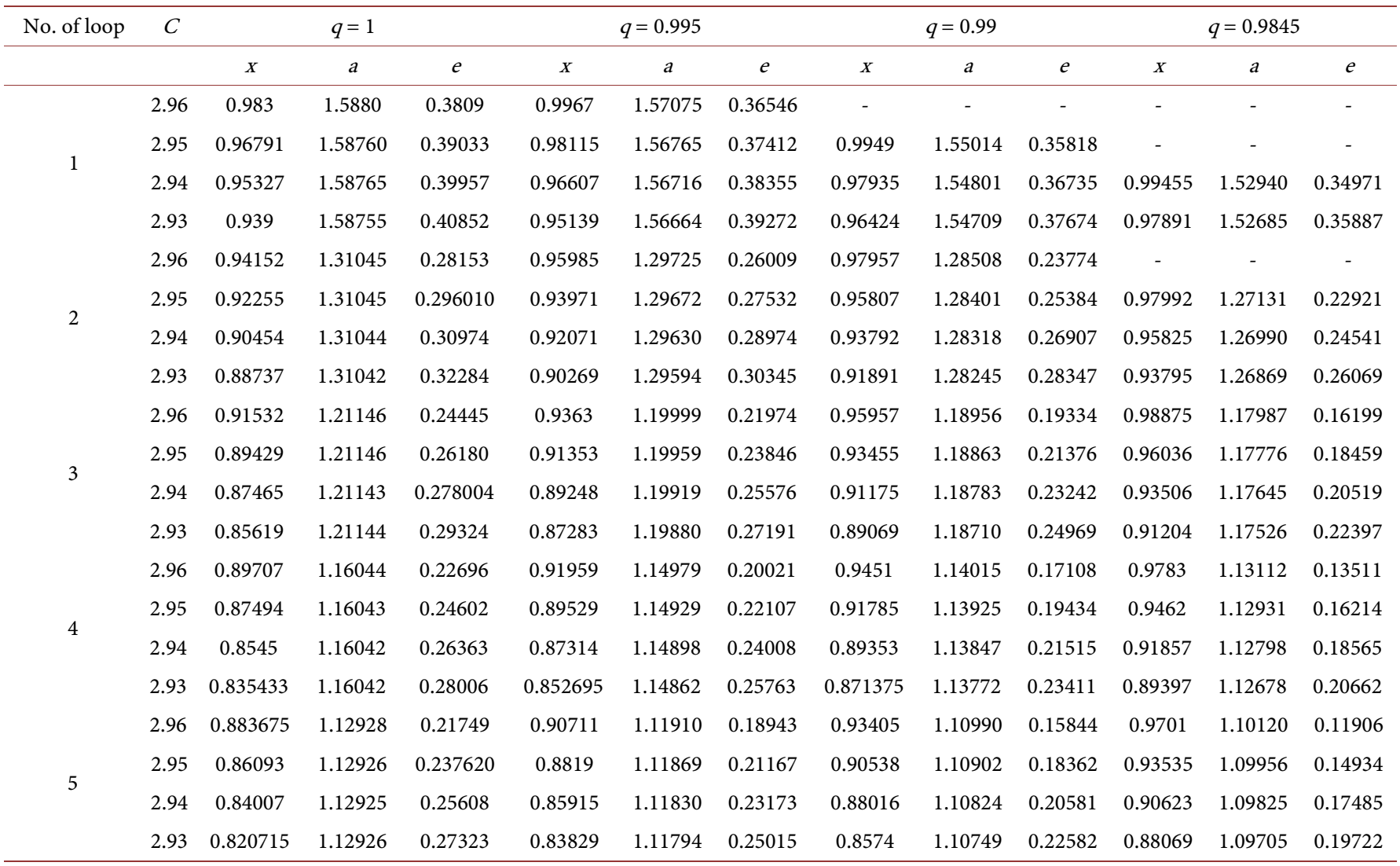


Table 6. Location, semi major axis and eccentricity of orbits for $q=1,0.995,0.99$ and 0.9845 for Sun-Earth system.

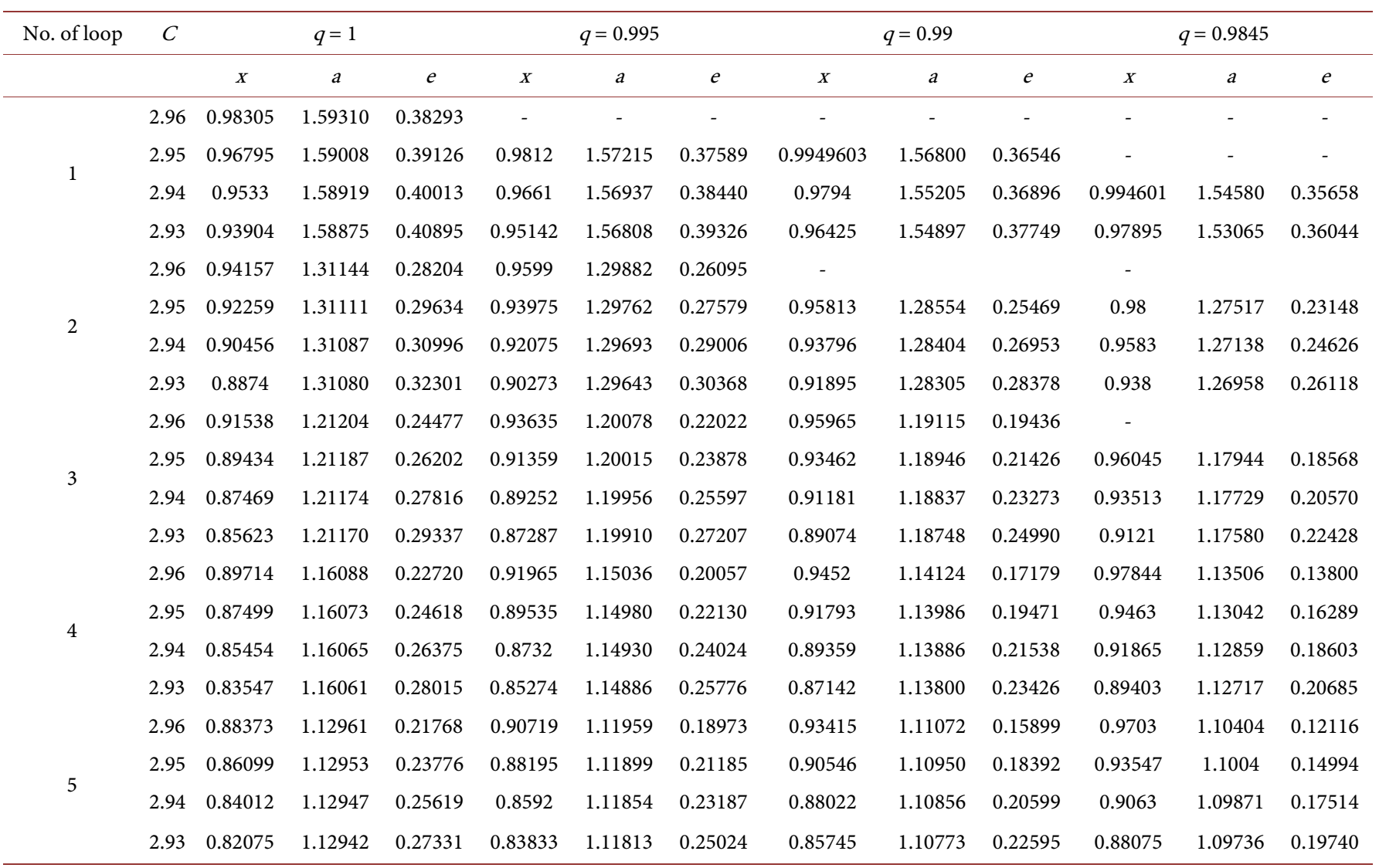

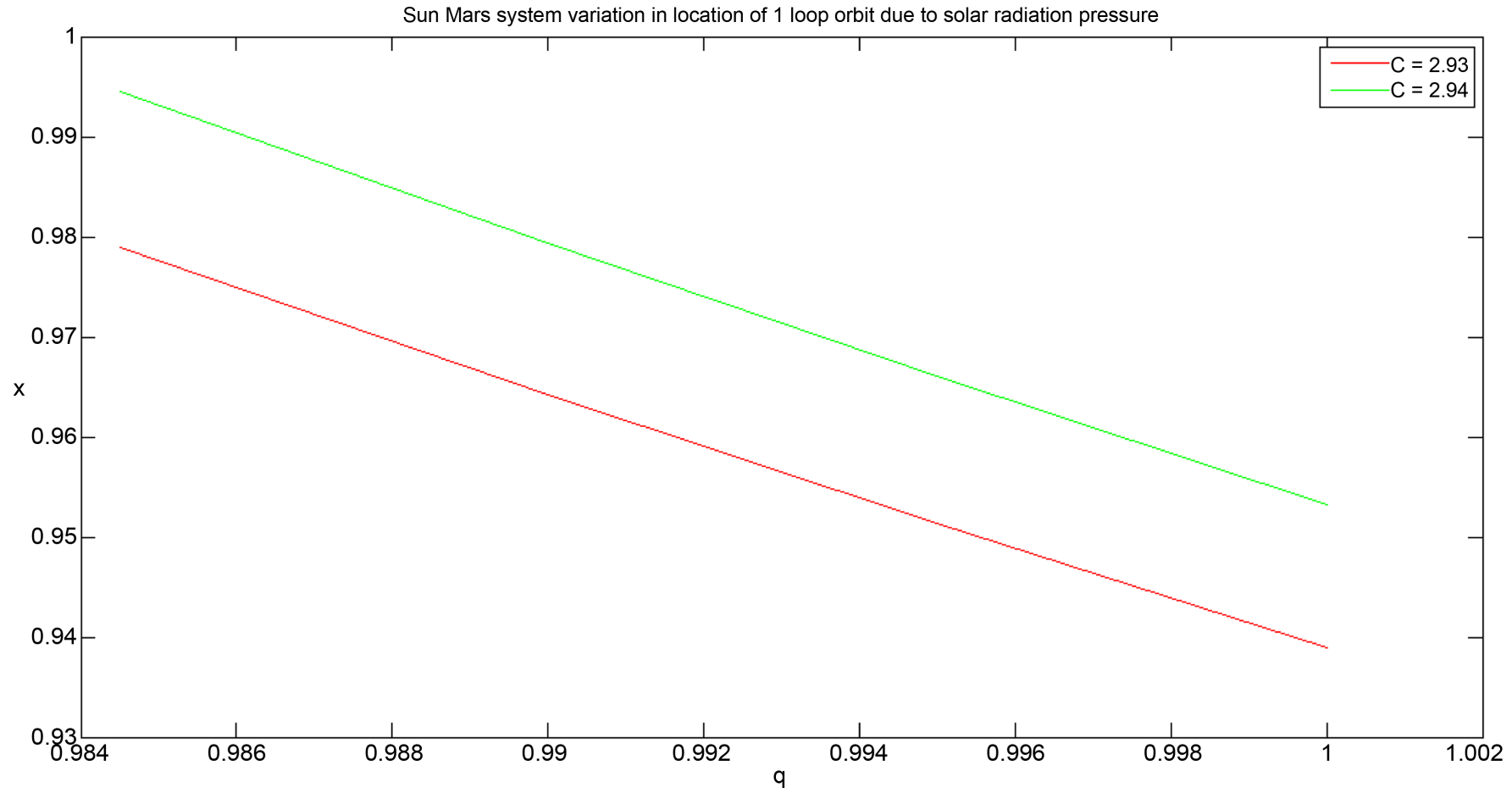

Figure 5. Variation in location of single loop periodic orbit around Sun-Mars system due to solar radiation pressure. 
observations for 5 loops closed periodic orbit in both Sun-Mars and Sun-Earth systems are shown in Figure 7 and Figure 8.

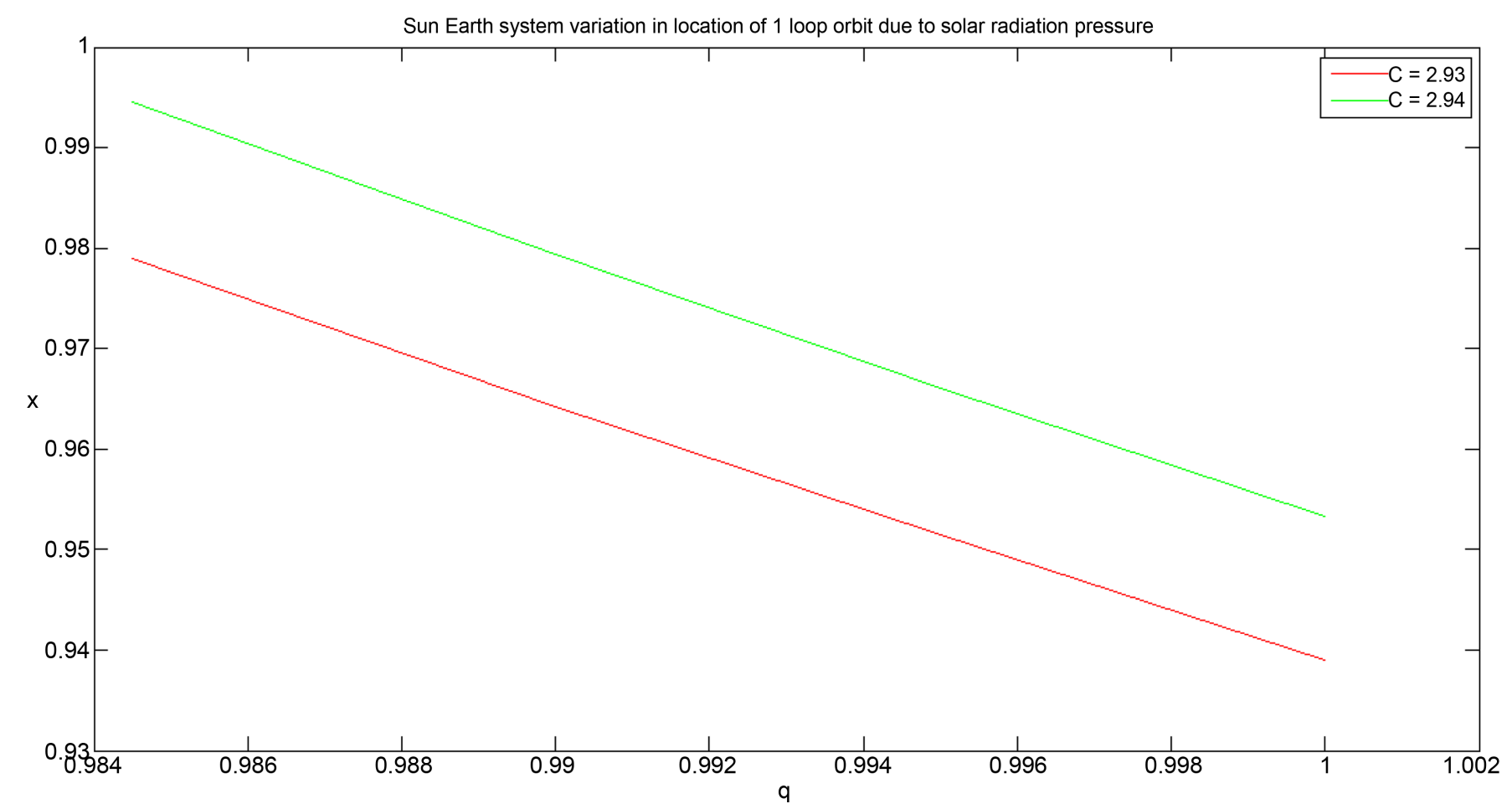

Figure 6. Variation in location of one loop periodic orbit around Sun-Earth due to solar radiation pressure.

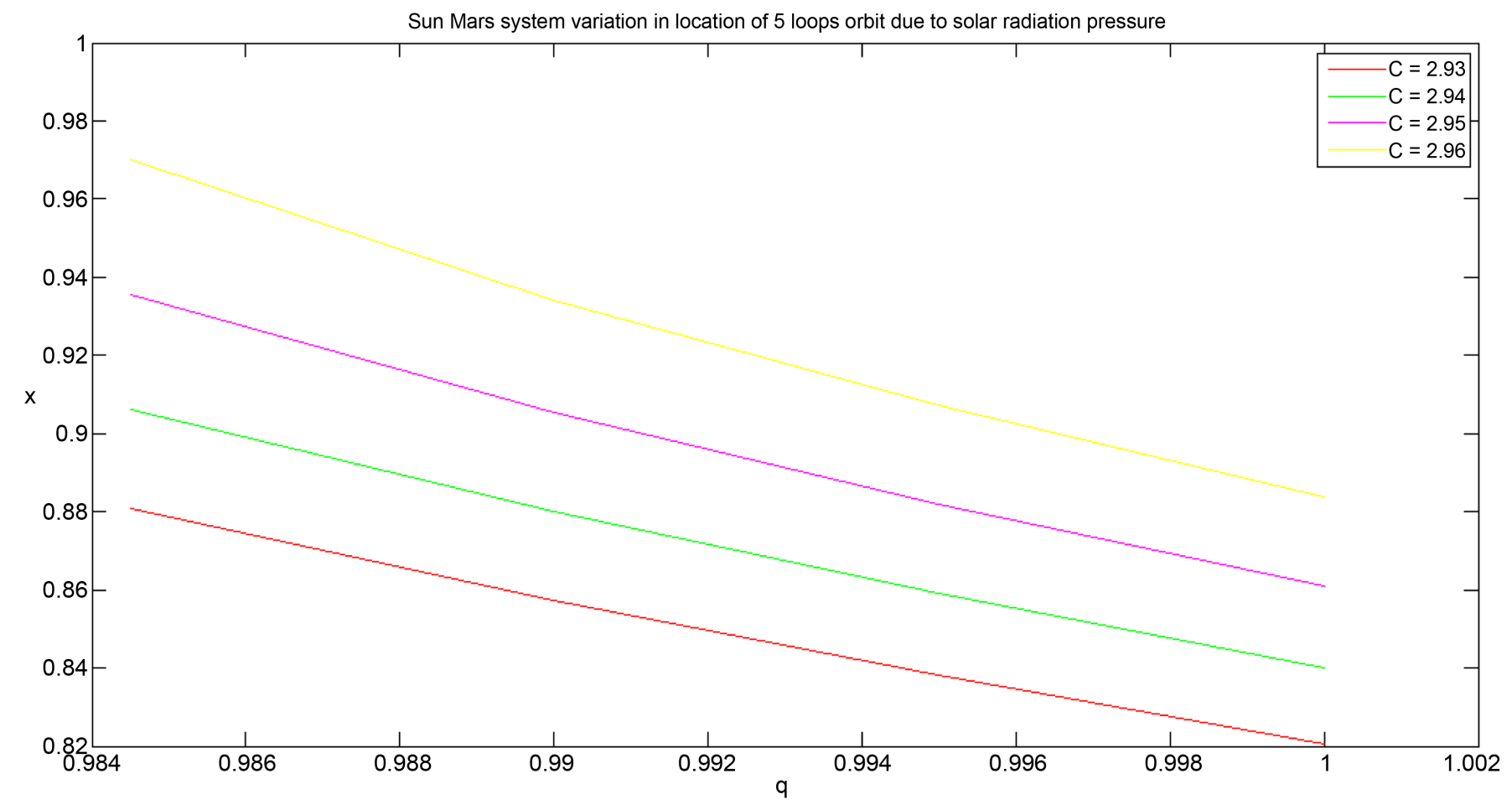

Figure 7. Variation in location of five loops periodic orbit around Sun-Mars system due to solar radiation pressure. 
From Figure 9 and Figure 10, it can be observed that for given solar radiation pressure, location of periodic orbit moves away from second primary as number of loops in

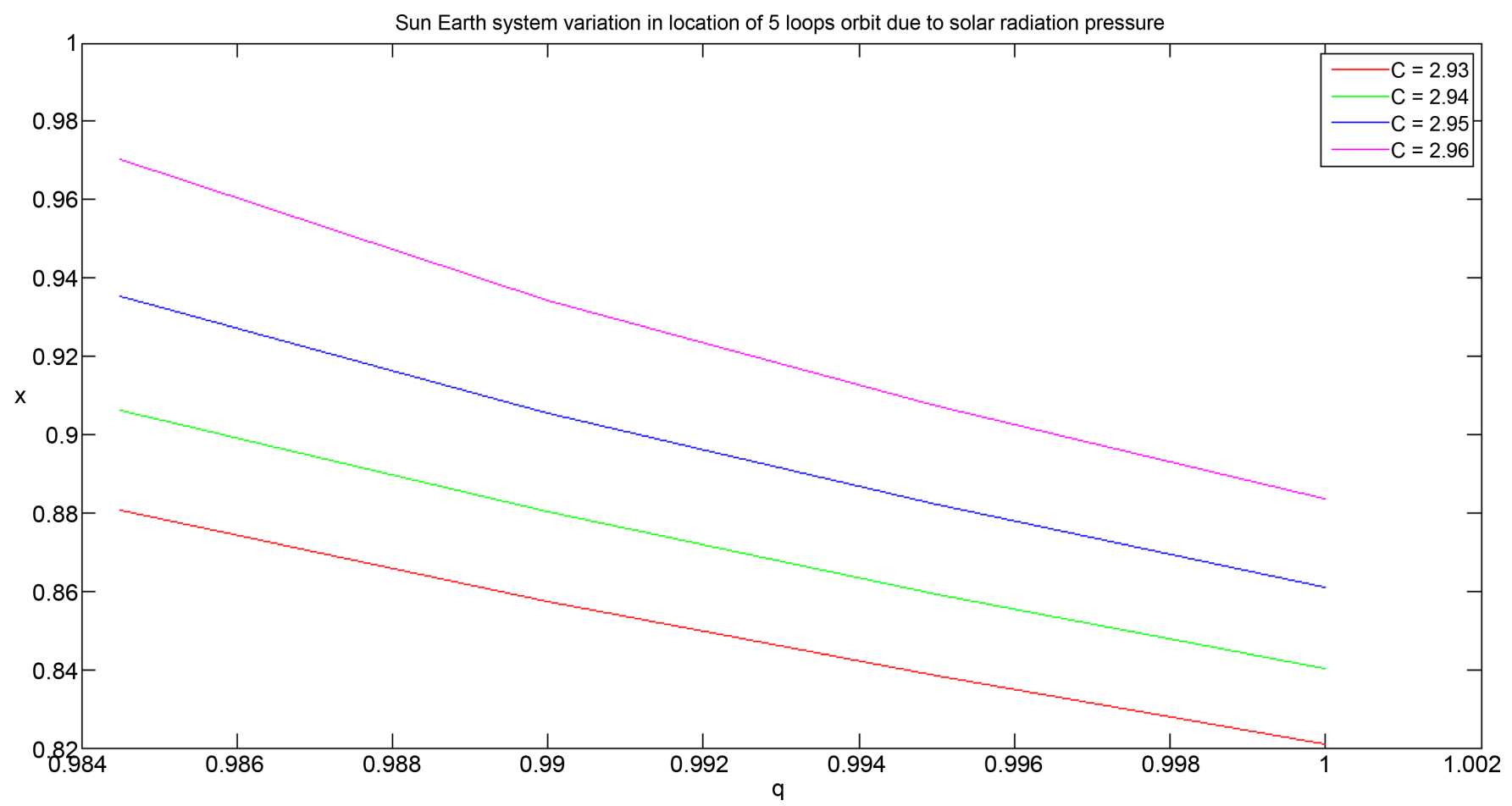

Figure 8. Variation in location of five loops periodic orbit around Sun-Earth system due to solar radiation pressure.

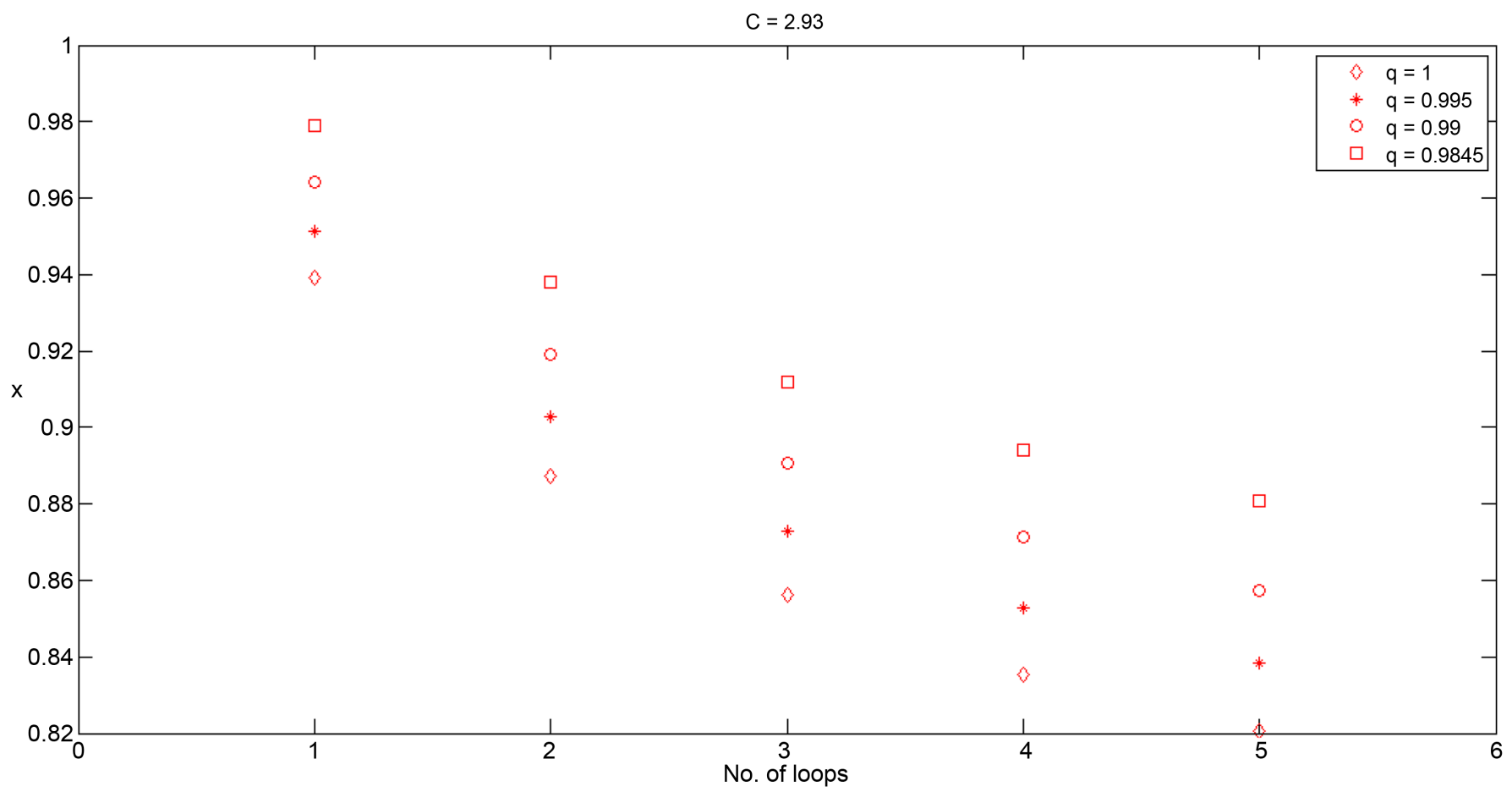

Figure 9. Variation in location of periodic orbit around Sun-Mars system for $C=2.93$ due to number of loops for different solar radiation pressure. 


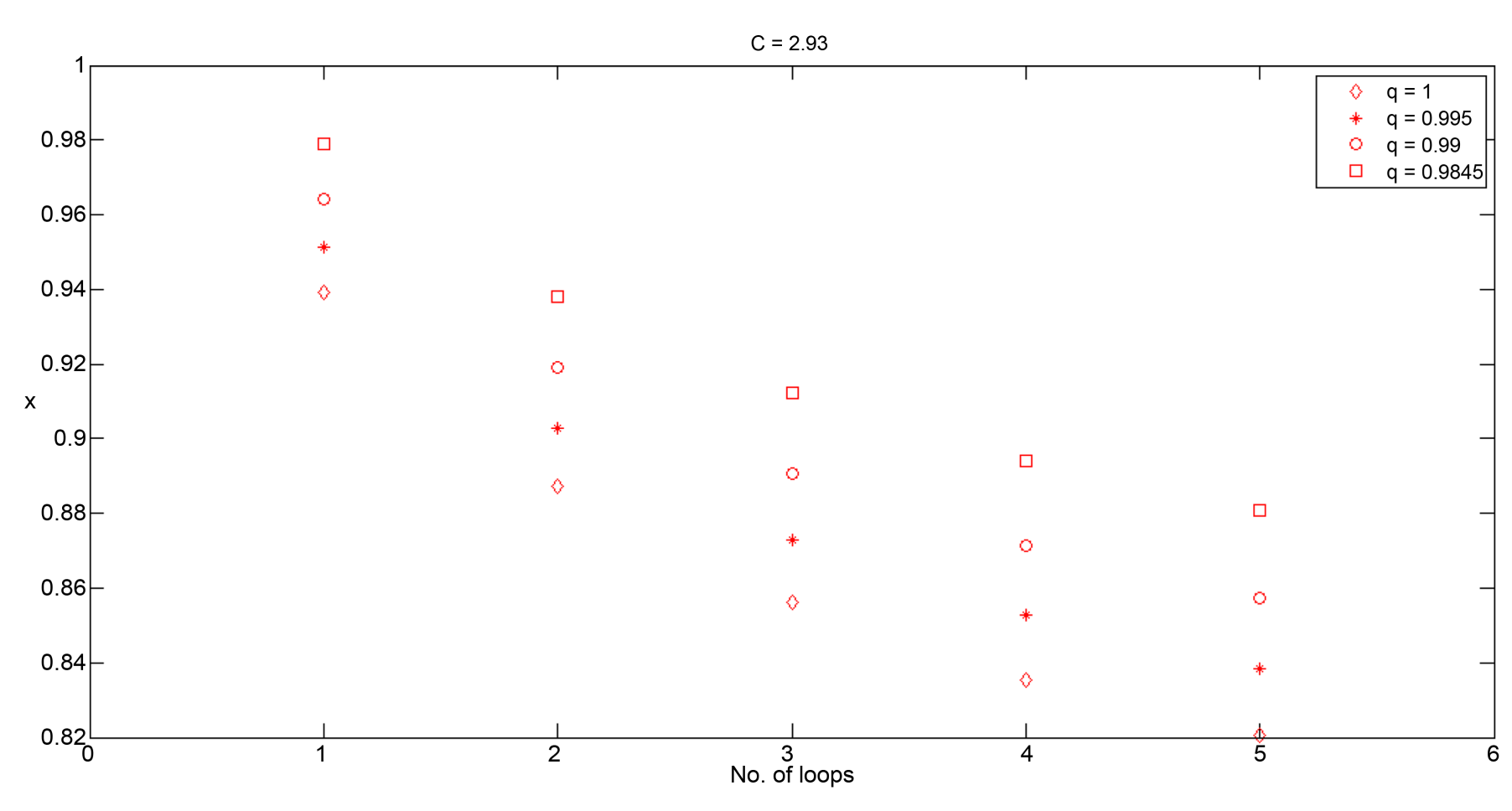

Figure 10. Variation in location of periodic orbit around Sun-Earth system for $C=2.93$ due to number of loops for different solar radiation pressure.

periodic orbit increase. Also, as solar radiation pressure increases from 1 to 0.9845 , location of periodic orbit moves towards second primary.

We have examined the effect of solar radiation pressure $q$ for different values of Jacobi constants $C$ on the semi major axis of periodic orbits with single loop and 5-loops of Sun-Mars and Sun-Earth systems. The results of these effects are displayed in Figures 11-14. While there are significant changes in the semi major axis due to solar radiation pressure, the effect of $C$ on the semi-major axis is less in comparison with that of q. Figures 11-14 indicate that the semi major axis decreases as solar radiation pressure increases from 1 to 0.9845 . Figure 12 shows behavior of semi major axis of single loop periodic orbit for different solar radiation pressure. Green curve indicates variation of semi major axis for $C=2.94$. It shows non smooth behavior near $q=0.99$, because single loop periodic orbit for $C=2.94$ and $q=0.9845$ is located at $x=0.994601$ loses its periodicity after time $t=110$. Figure 14 shows behavior of semi major axis of five loops periodic orbits for different $q$. Magenta curve indicates variation for semi major axis for $C=2.96$. It shows non smooth behavior near $q=0.99$, because five loops periodic orbit for $C=2.96$ and $q=0.9845$ is located at $x=0.9703$ loses its periodicity after time $t=$ 500 .

The variation of semi major axis against the number of loops is examined for different solar radiation pressure and the results of the study are displayed in Figure 15 and Figure 16. The semi major axis decreases with increase in the number of loops. Further for a fixed number of loops, the semi-major axes decreases with increase in solar radiation pressure from 1 to 0.9845 . 


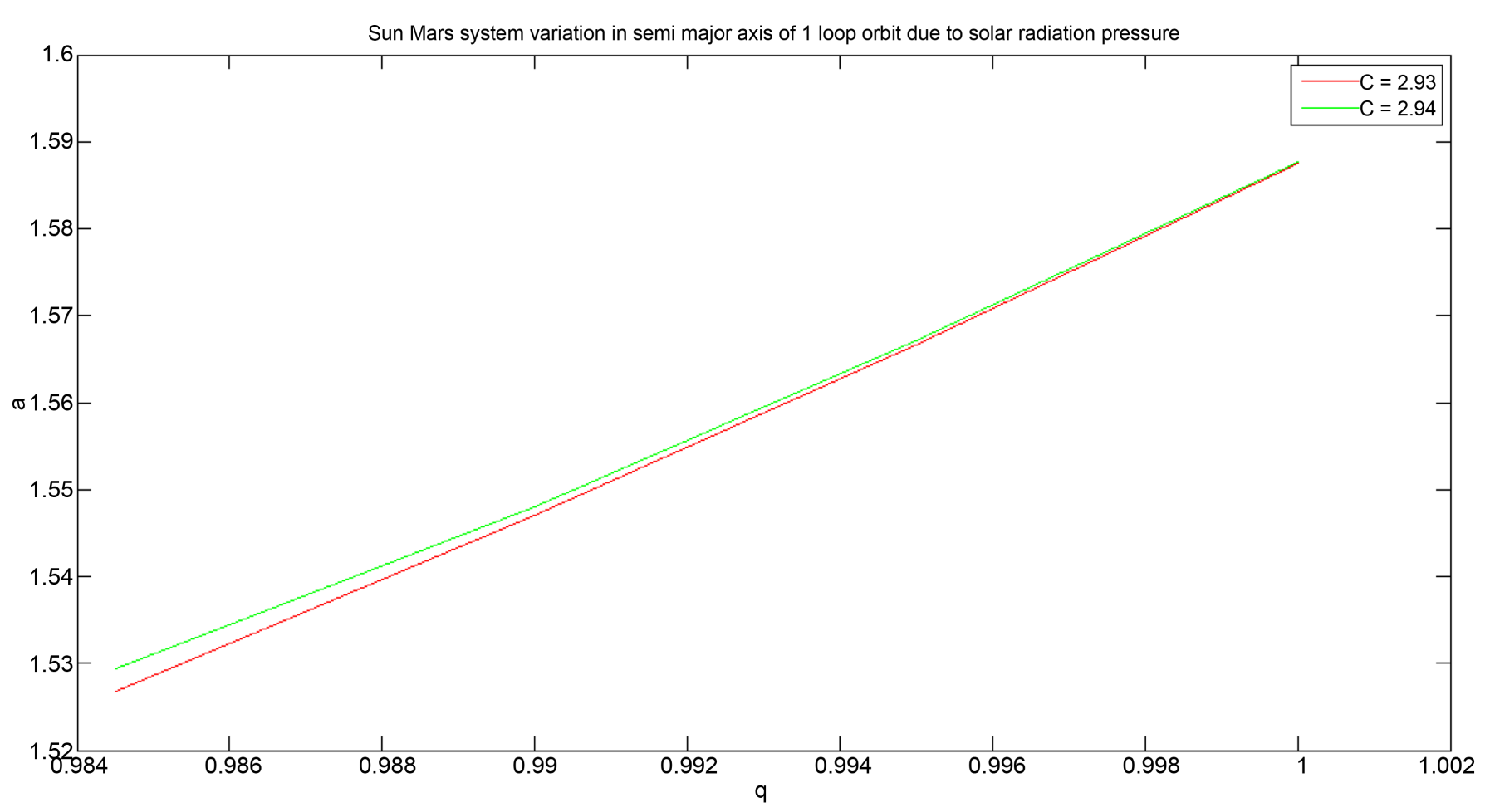

Figure 11. Variation in semi major axis of one loop periodic orbit around Sun-Mars system due to solar radiation pressure.

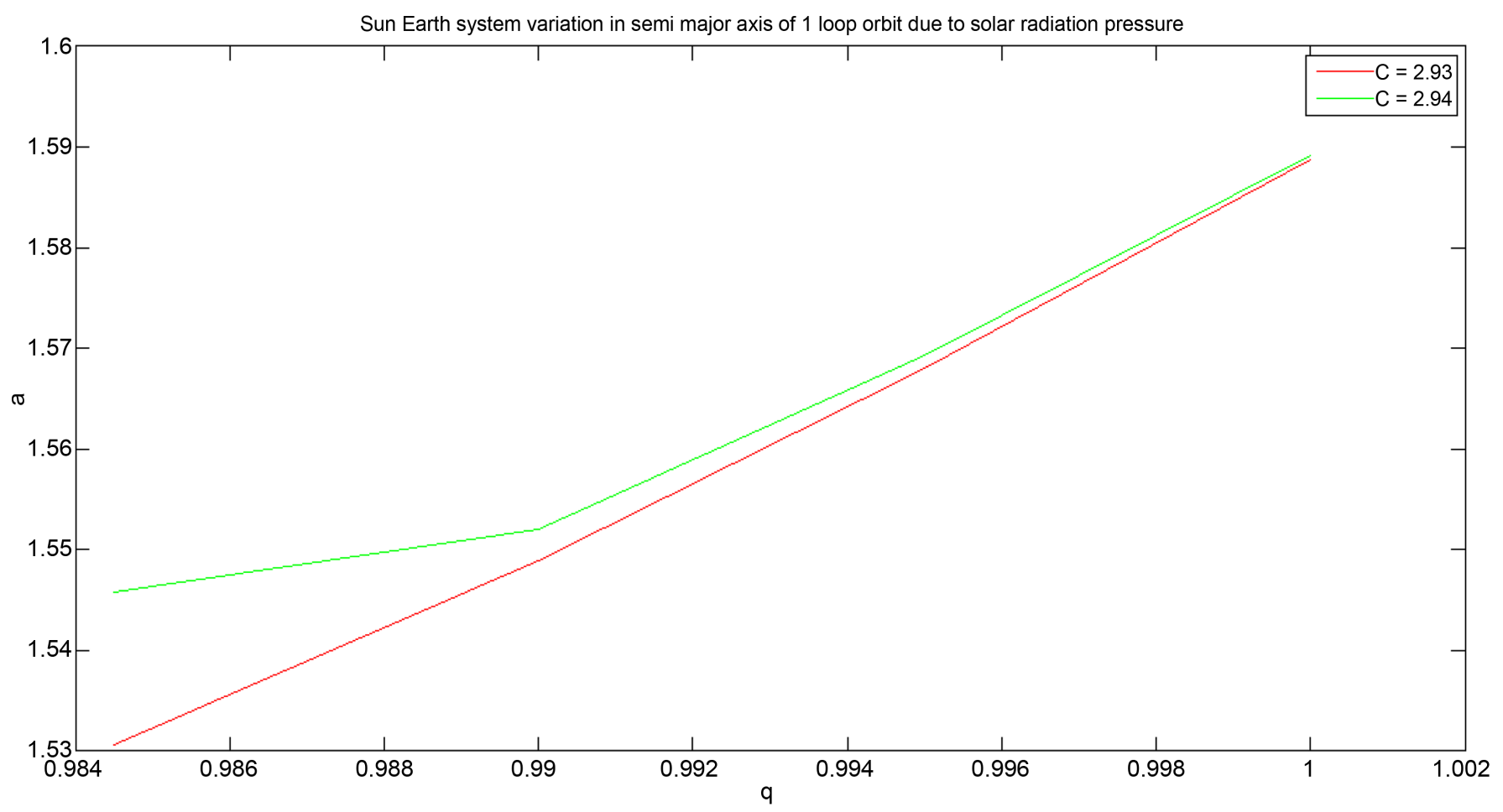

Figure 12. Variation in semi major axis of one loop periodic orbit around Sun-Earth system due to solar radiation pressure.

From Figure 15 and Figure 16 it can be observed that, for given pair of $C$ and $q$, as the number of loops increases in periodic orbit, semi major axis decreases. Also, as solar 


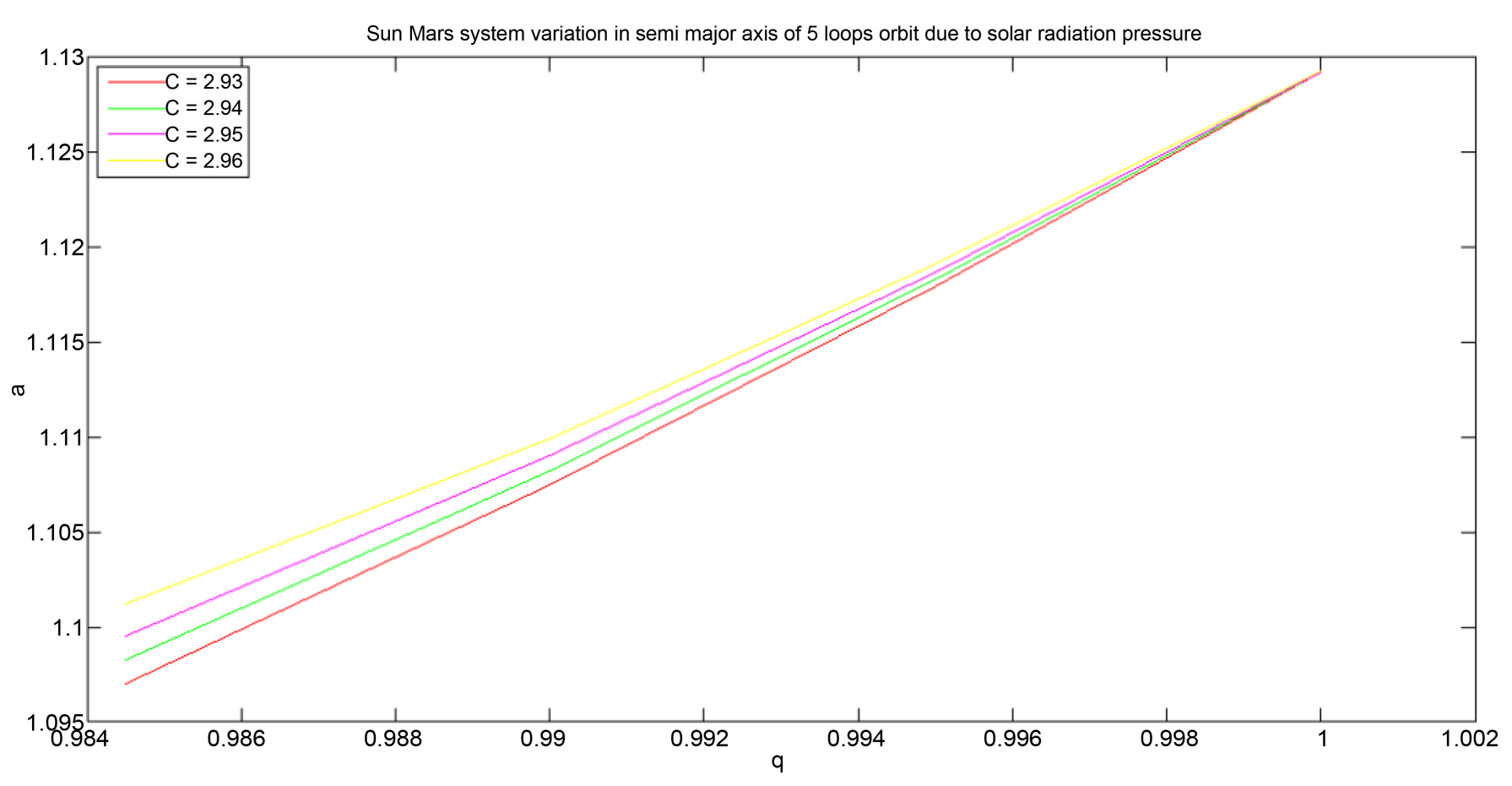

Figure 13. Variation in semi major axis of five loops periodic orbit around Sun-Mars system due to solar radiation pressure.

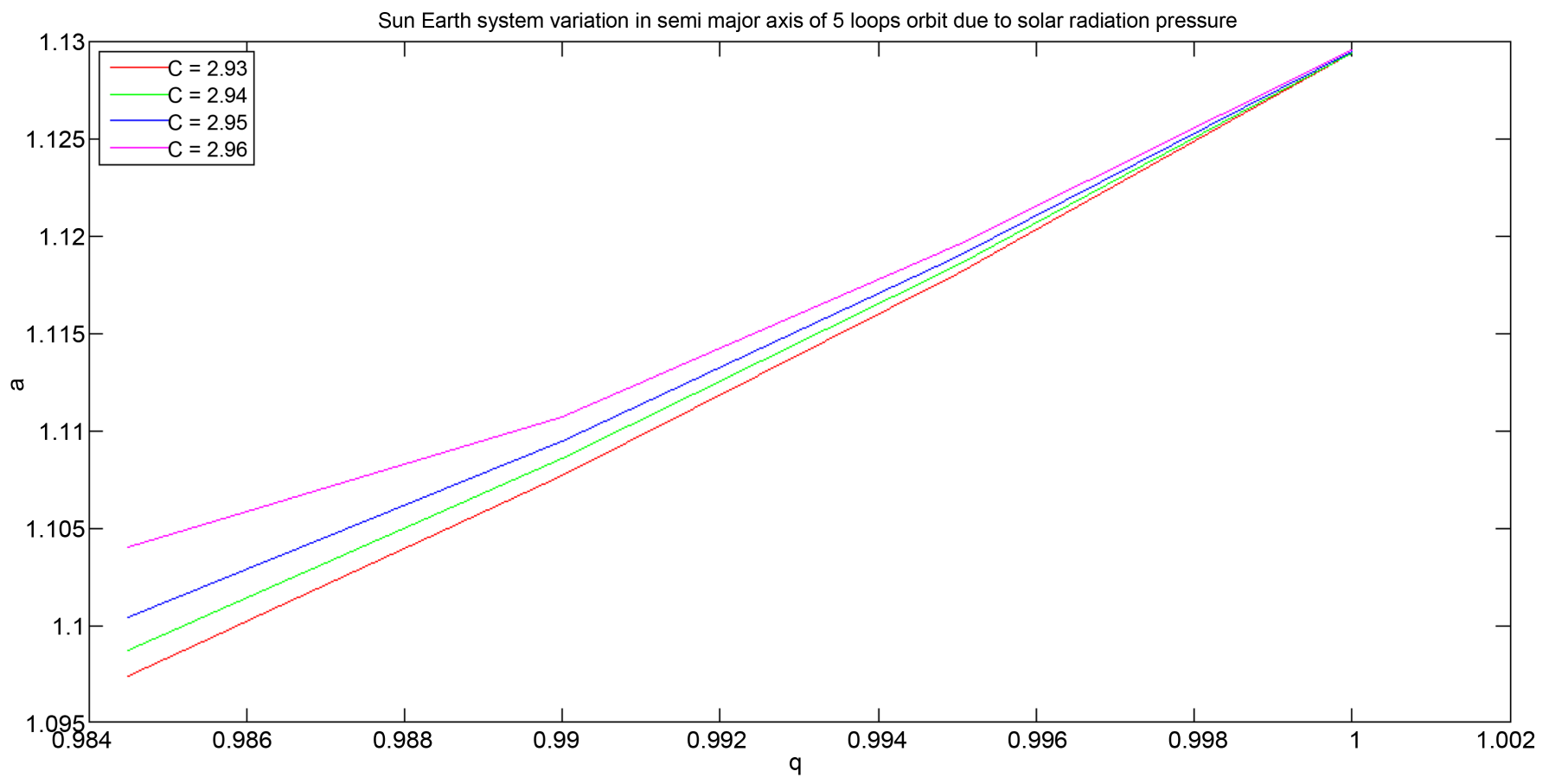

Figure 14. Variation in semi major axis of five loops periodic orbit around Sun-Earth system due to solar radiation pressure.

radiation pressure increases from 1 to 0.9845 semi major axis of periodic orbit for given loop decreases.

The variations of the other geometric parameter, viz, eccentricity $e$ with respect to solar radiation pressure $q$ and Jacobi constant $C$ for single and five loops periodic orbits 


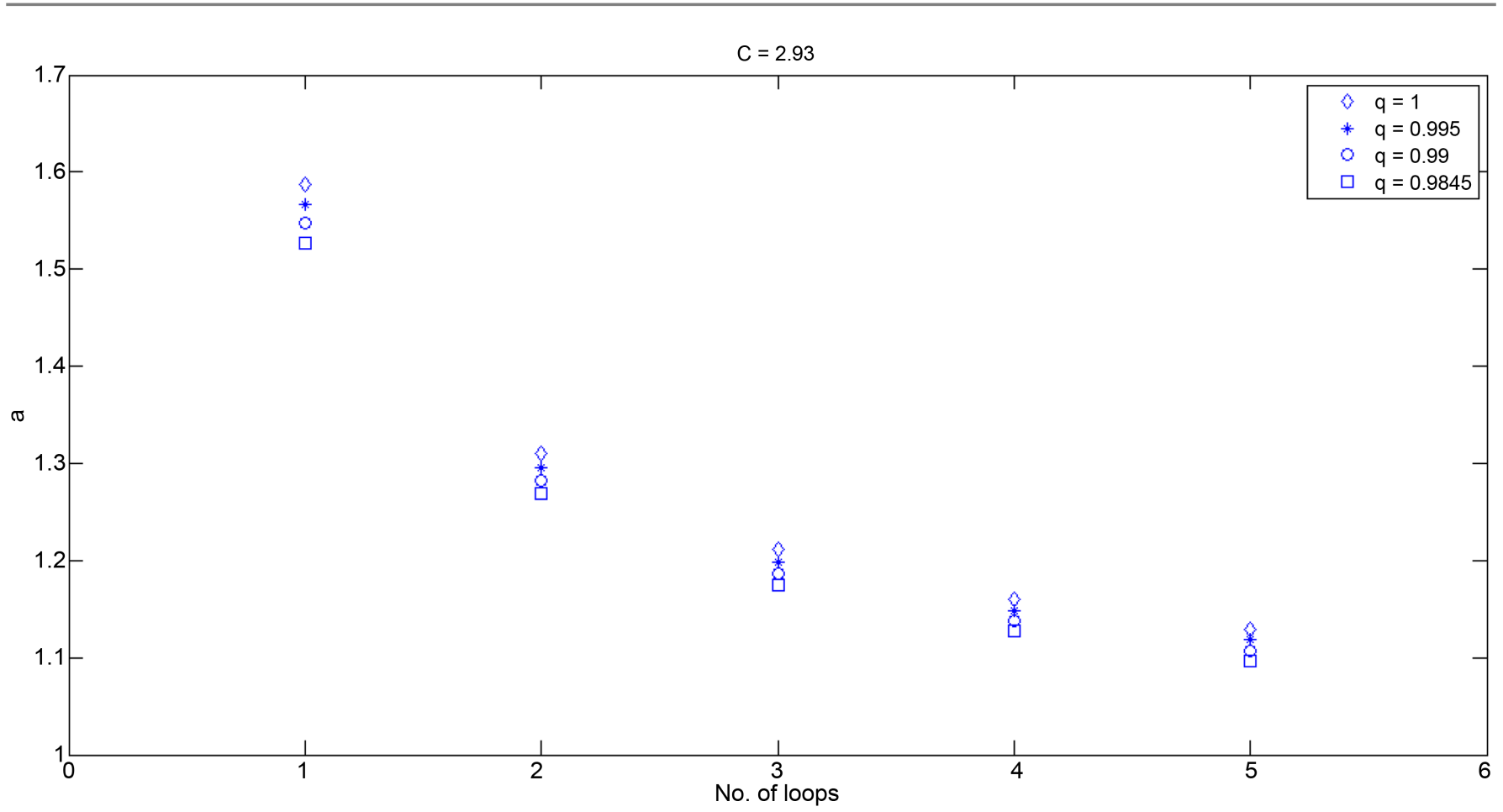

Figure 15. Variation in semi major axis of periodic orbit around Sun-Mars system due to number of loops for $C=2.93$.

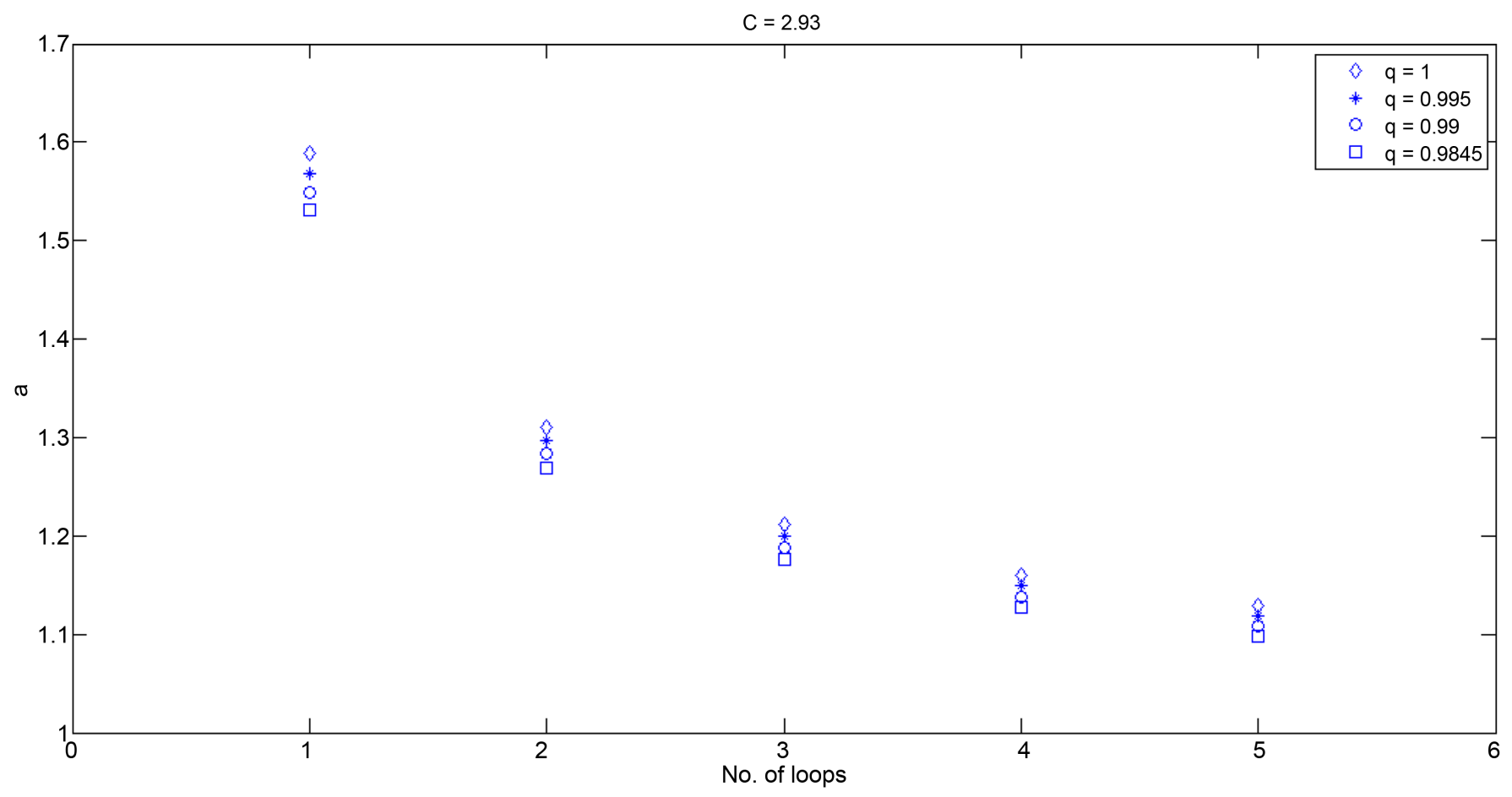

Figure 16. Variation in semi major axis of periodic orbit around Sun-Earth system due to number of loops.

are shown in Figures 17-20 for both Sun-Mars and Sun-Earth systems. The eccentricity decreases as $q$ increases from 1 to 0.9845 and decreases as $C$ increases for single loop periodic orbit for both systems Sun-Mars and Sun-Earth as shown in Figure 17 and 


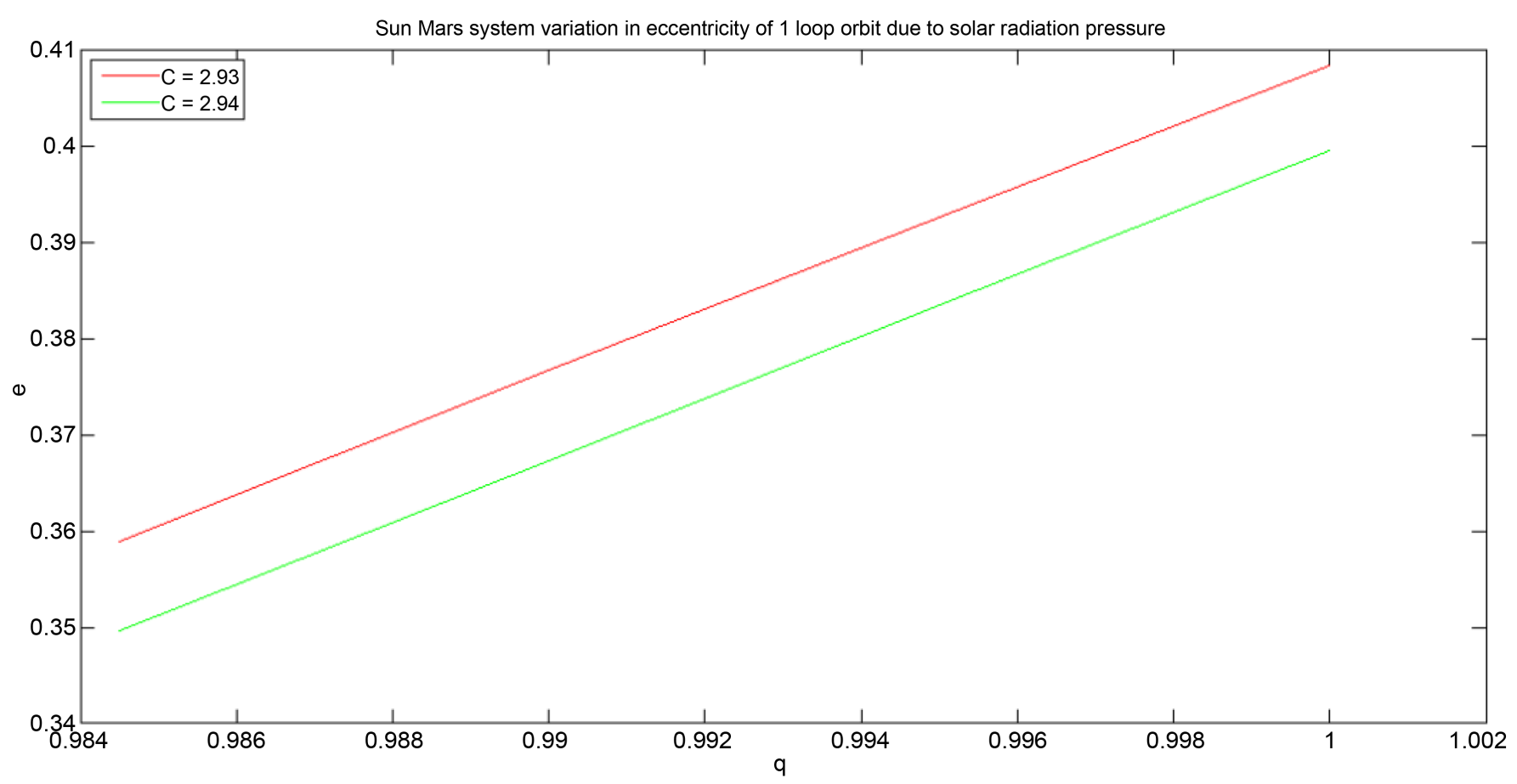

Figure 17. Variation in eccentricity of single loop periodic orbit around Sun-Mars system due to solar radiation pressure.

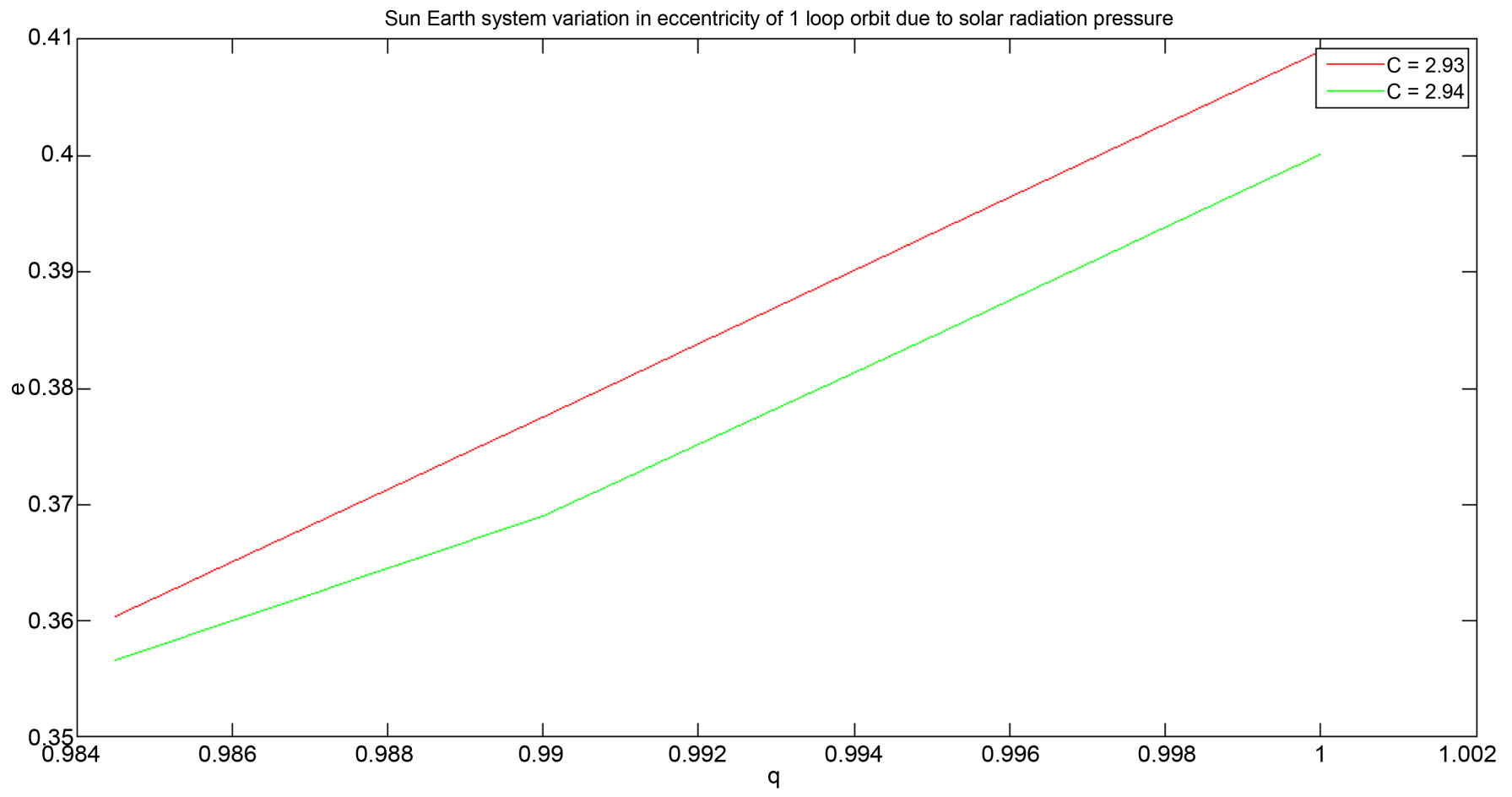

Figure 18. Variation in eccentricity of single loop periodic orbit around Sun and Earth due to oblateness.

Figure 18. Here we can observe that effect of orbits which lost their periodicity after some time is less on eccentricity of the periodic orbit in comparison of semi major axis of the periodic orbit. Figure 18 shows variation in eccentricity of single loop orbit 


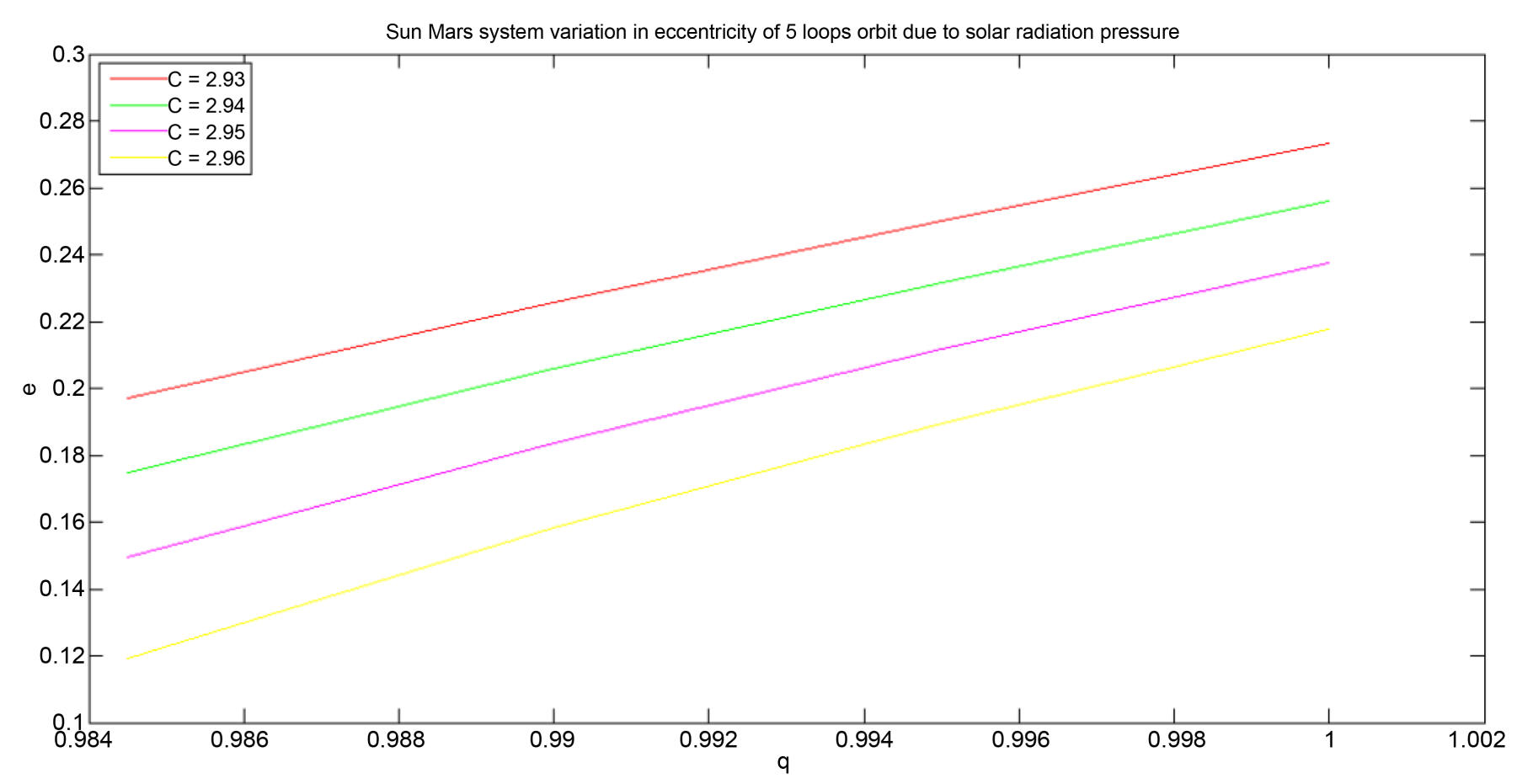

Figure 19. Variation in eccentricity of five loops periodic orbit around Sun-Mars system due to solar radiation pressure.

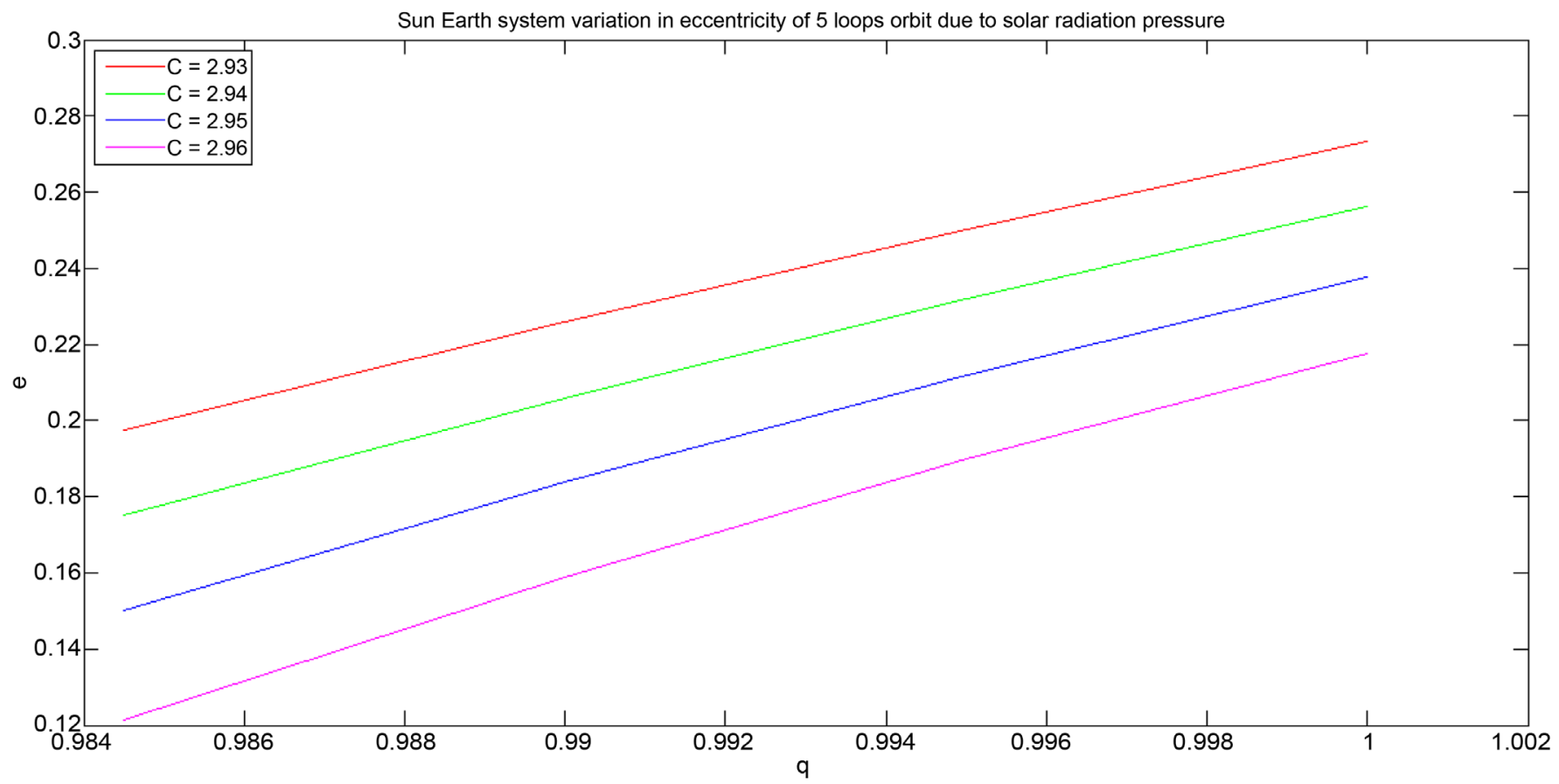

Figure 20. Variation in eccentricity of five loops periodic orbit around Sun-Earth system due to solar radiation pressure.

corresponding to variation in $\mathrm{q}$ for given $C$. green curve shows non smooth behavior corresponding to periodic orbit for $C=2.94, q=0.9845$ which is located at $x=0.994601$, loses its periodicity after $t=110$.

We have also analyzed the effect of number of loops on the eccentricity of the orbit. 
These effects are depicted in Figure 21 and Figure 22. It is found that the eccentricity decreases as number of loops increases. For a given loop, the eccentricity is found to decrease as solar radiation pressure increases 1 to 0.9845 .

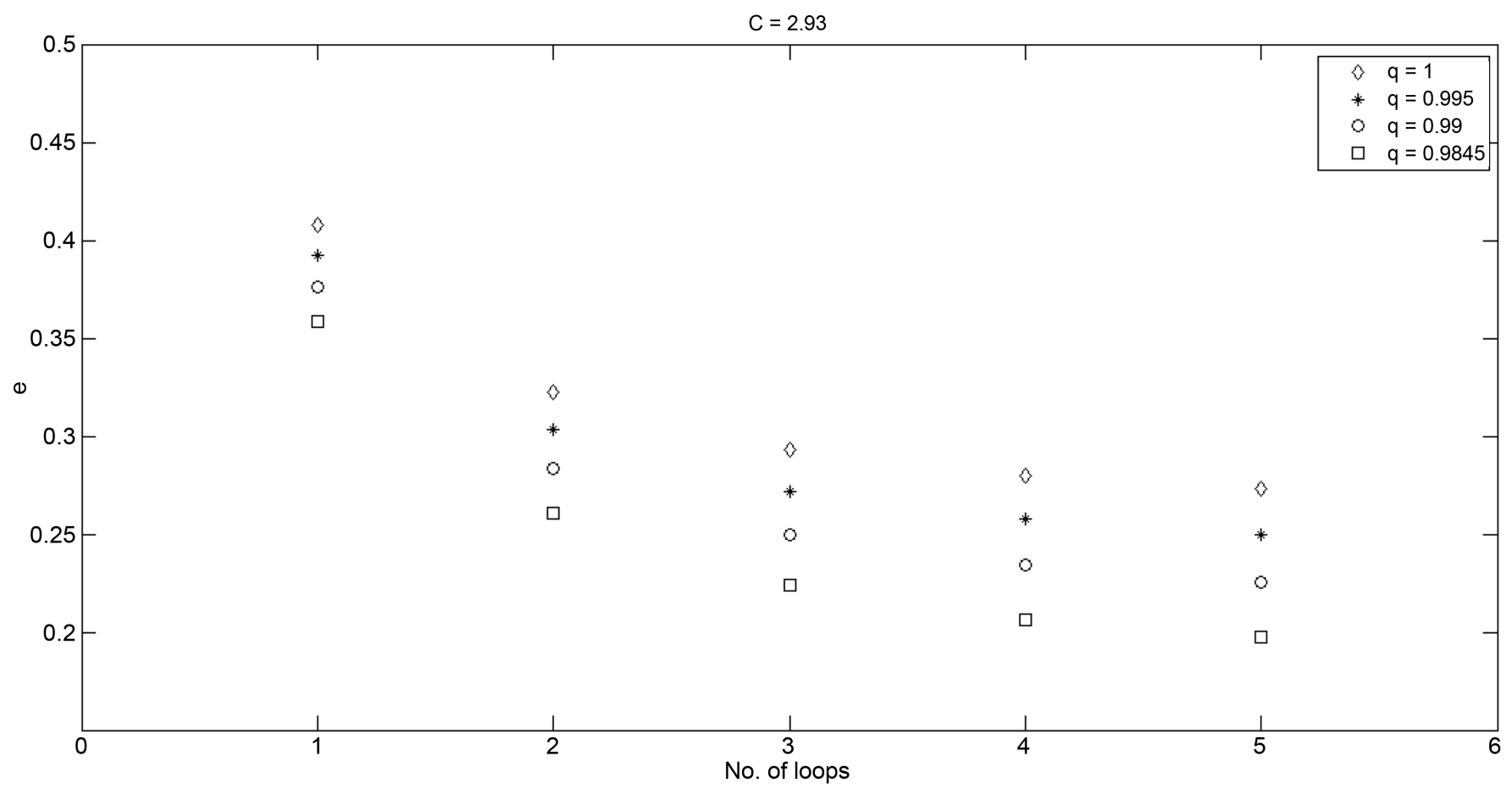

Figure 21. Variation in eccentricity of periodic orbit around Sun-Mars system due to number of loops.

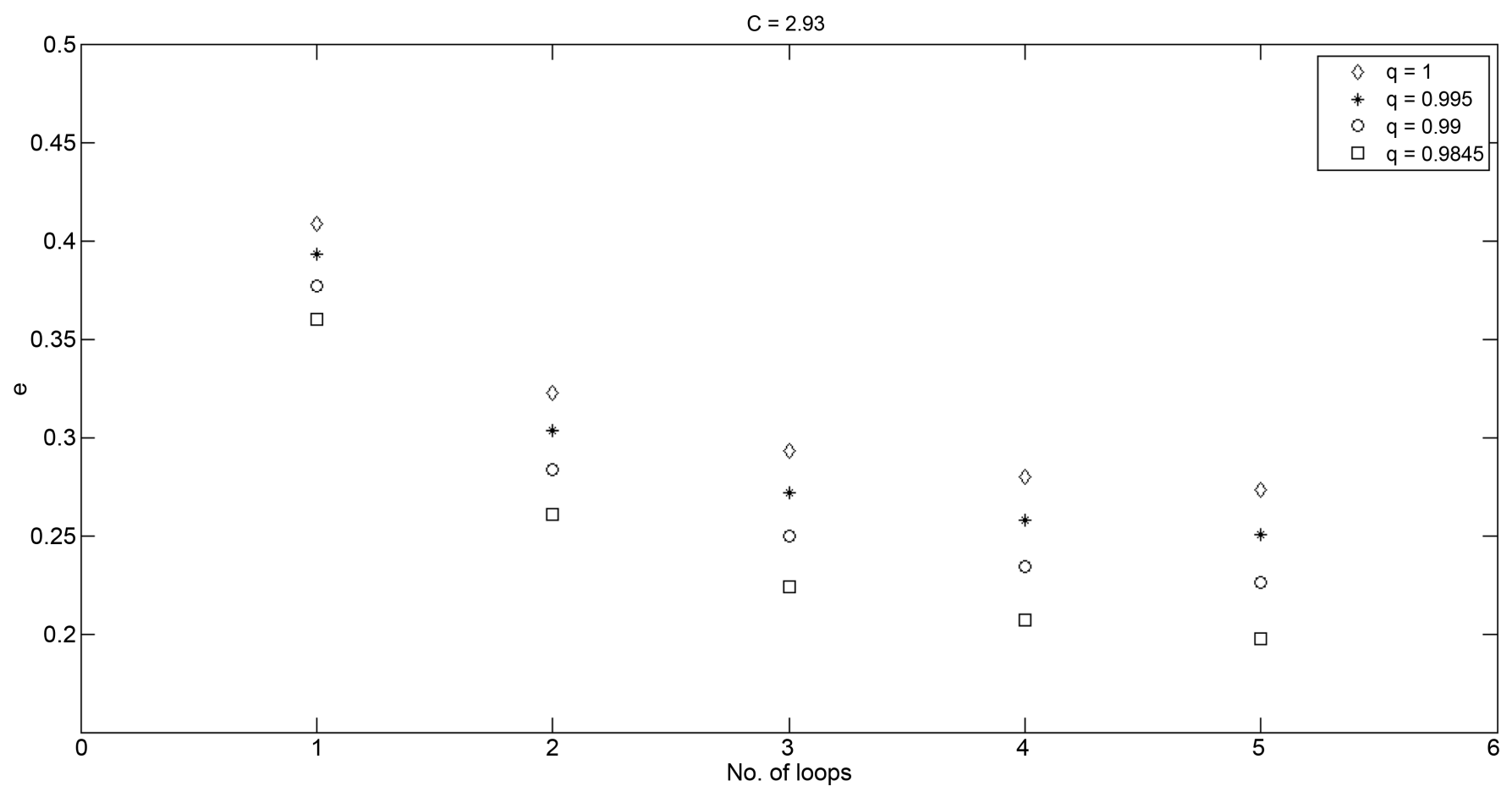

Figure 22. Variation in eccentricity of periodic orbit around Sun and Earth due to number of loops. 
In Table 5 initial velocity of secondary body is denoted by $V, D_{1}$ and $D_{2}$ are the distance of secondary body from Mars and Sun respectively. $V$ is in $\mathrm{kms}^{-1}, D_{1}$ and $D_{2}$ are in $\mathrm{km}$. Similar notations are used in Table 6 also. In Table 6 distance of secondary body from Earth is denoted by $D_{1} . V$ can be obtained using Equation (11).

The conversion from units of distance $(I)$ and velocity $(/)$ in the normalized dimension less system to the dimensionalized system is given by,

$$
\begin{aligned}
& \text { Distance } D=L \times I \text {, } \\
& \text { Velocity } V=O \times J \text {. }
\end{aligned}
$$

where $L$ is the distance between the centers of both primaries in $\mathrm{km}$. $O$ is the orbital velocity of second primary around first primary [12]. For Sun Mars and Sun Earth system $L=227,940,000$ and $149,600,000 \mathrm{~km}$ respectively. Mean orbital velocity of Mars around Sun and Earth around Sun are $24.07 \mathrm{~km} \cdot \mathrm{sec}^{-1}$ and $29.78 \mathrm{~km} \cdot \mathrm{sec}^{-1}$ respectively.

It can be observed from Table 7 and Table 8 that for given solar radiation pressure and given loop orbit as $C$ decreases, $V$ and $D_{1}$ increases and $D_{2}$ decreases. For given $C$ and given number of loop as $q$ increases from 1 to 0.9845 initial velocity $V$ and $D_{1}$ decreases and $D_{2}$ increases. So, the effect of Jacobi constant $C$ and solar radiation pressure

\begin{tabular}{|c|c|c|c|c|c|c|c|c|c|c|c|c|c|c|c|c|c|}
\hline \multirow[t]{3}{*}{ No. of loop } & \multirow[t]{3}{*}{$C$} & \multicolumn{4}{|c|}{$q=1$} & \multicolumn{4}{|c|}{$q=0.995$} & \multicolumn{4}{|c|}{$q=0.99$} & \multicolumn{4}{|c|}{$q=0.9845$} \\
\hline & & & V & $D_{1}$ & & & \multirow{2}{*}{$\begin{array}{c}V \\
\left(\mathrm{~km} \cdot \mathrm{sec}^{-}\right.\end{array}$} & $D_{1}$ & & \multirow{2}{*}{ ( } & \multirow{2}{*}{$\begin{array}{c}V \\
\left(\mathrm{~km} \cdot \mathrm{sec}^{-}\right.\end{array}$} & $D_{1}$ & $D_{2}$ & \multirow{2}{*}{$X$} & \multirow{2}{*}{$\begin{array}{c}V \\
\left(\mathrm{~km} \cdot \mathrm{sec}^{-1}\right.\end{array}$} & \multirow[t]{2}{*}{$D_{1}$} & $D_{2}$ \\
\hline & & & $\left(\mathrm{km} \cdot \mathrm{sec}^{-1}\right.$ & $\left(10^{\prime} \mathrm{km}\right)$ & $\left(10^{\circ} \mathrm{km}\right)$ & & & $\left(10^{\prime} \mathrm{km}\right)$ & $\left(10^{\circ} \mathrm{km}\right)$ & & & & & & & & \\
\hline \multirow{4}{*}{1} & 2.96 & 0.983 & 28.52 & 0.38749 & 2.24065 & 0.9967 & 28.17 & 0.07521 & 2.27187 & - & - & - & - & - & - & - & - \\
\hline & 2.95 & 0.96791 & 28.84 & 0.73145 & 2.20625 & 0.98115 & 28.48 & 0.42965 & 2.23643 & 0.9949 & 28.12 & 0.11624 & 2.26777 & - & - & - & - \\
\hline & 2.94 & 0.95327 & 29.16 & 1.06515 & 2.17288 & 0.96607 & 28.80 & 0.77339 & 2.20206 & 0.97935 & 28.44 & 0.47068 & 2.23233 & 0.99455 & 28.04 & 0.12421 & 2.26697 \\
\hline & 2.93 & 0.939 & 29.47 & 1.39042 & 2.14035 & 0.95139 & 29.12 & 1.10800 & 2.16859 & 0.96424 & 28.76 & 0.81510 & 2.19788 & 0.97891 & 28.35 & 0.48071 & 2.23132 \\
\hline \multirow{4}{*}{2} & 2.96 & 0.94152 & 28.08 & 1.33298 & 2.14610 & 0.95985 & 27.57 & 0.91517 & 2.18788 & 0.97957 & 27.05 & 0.46567 & 2.23283 & - & - & - & - \\
\hline & 2.95 & 0.92255 & 28.52 & 1.76538 & 2.10286 & 0.93971 & 28.04 & 1.37424 & 2.14197 & 0.95807 & 27.53 & 0.95574 & 2.18382 & 0.97992 & 26.95 & 0.45769 & 2.23363 \\
\hline & 2.94 & 0.90454 & 28.96 & 2.17590 & 2.06180 & 0.92071 & 28.48 & 1.80732 & 2.09866 & 0.93792 & 27.99 & 1.41504 & 2.13789 & 0.95825 & 28.05 & 0.95164 & 2.18423 \\
\hline & 2.93 & 0.88737 & 29.38 & 2.56728 & 2.02267 & 0.90269 & 28.92 & 2.21807 & 2.05759 & 0.91891 & 28.44 & 1.84835 & 2.09456 & 0.93795 & 27.90 & 1.41436 & 2.13796 \\
\hline & 2.96 & 0.91532 & 28.06 & 1.93018 & 2.08638 & 0.9363 & 27.47 & 1.45197 & 2.13420 & 0.95957 & 26.84 & 0.92155 & 2.18724 & 0.98875 & 26.09 & 2.56425 & 2.25375 \\
\hline & 2.95 & 0.89429 & 28.59 & 2.40954 & 2.03844 & 0.91353 & 28.02 & 1.97098 & 2.08230 & 0.93455 & 27.43 & 1.49185 & 2.13021 & 0.96036 & 26.73 & 0.90354 & 2.18904 \\
\hline & 2.94 & 0.87465 & 29.09 & 2.85722 & 1.99367 & 0.89248 & 28.55 & 2.45080 & 2.03431 & 0.91175 & 27.98 & 2.01156 & 2.07824 & 0.93506 & 27.32 & 1.48023 & 2.13137 \\
\hline & 2.93 & 0.85619 & 29.58 & 3.27799 & 1.95160 & 0.87283 & 29.05 & 2.89870 & 1.98952 & 0.89069 & 28.51 & 2.49160 & 2.03023 & 0.91204 & 27.88 & 2.00495 & 2.07890 \\
\hline \multirow{4}{*}{4} & 2.96 & 0.89707 & 28.14 & 2.34617 & 2.04478 & 0.91959 & 27.49 & 1.83285 & 2.09611 & 0.9451 & 26.79 & 1.25138 & 2.15426 & 0.9783 & 25.92 & 4.94622 & 2.22993 \\
\hline & 2.95 & 0.87494 & 28.72 & 2.85061 & 1.99433 & 0.89529 & 28.12 & 2.45080 & 2.03431 & 0.91785 & 27.45 & 1.87251 & 2.09214 & 0.9462 & 26.67 & 1.22630 & 2.15676 \\
\hline & 2.94 & 0.8545 & 29.27 & 3.31651 & 1.94774 & 0.87314 & 28.68 & 2.89163 & 1.99023 & 0.89353 & 28.06 & 2.42686 & 2.03671 & 0.91857 & 27.34 & 1.85610 & 2.09378 \\
\hline & 2.930 & .835433 & 29.79 & 3.75113 & 1.904280 & 0.852695 & 29.23 & 3.35766 & 1.94363 & 0.871375 & 28.64 & 2.93187 & 1.98621 & 0.89397 & 27.96 & 2.41684 & 2.03771 \\
\hline \multirow{4}{*}{5} & 2.960 & .883675 & 28.25 & 2.65150 & 2.01424 & 0.90711 & 27.56 & 2.11732 & 2.06766 & 0.93405 & 26.80 & 1.50325 & 2.12907 & 0.9701 & 25.85 & 6.81533 & 2.21124 \\
\hline & 2.95 & 0.86093 & 28.85 & 3.16995 & 1.96240 & 0.8819 & 28.11 & 2.38675 & 2.04072 & 0.90538 & 27.52 & 2.15676 & 2.06372 & 0.93535 & 26.68 & 1.47362 & 2.13203 \\
\hline & 2.94 & 0.84007 & 29.43 & 3.64543 & 1.91485 & 0.85915 & 28.82 & 3.21052 & 1.95834 & 0.88016 & 28.17 & 2.73162 & 2.00623 & 0.90623 & 27.40 & 2.13738 & 2.06566 \\
\hline & 2.930 & .820715 & 29.98 & 4.08672 & 1.87072 & 0.83829 & 29.39 & 3.68601 & 1.91079 & 0.8574 & 28.78 & 3.25041 & 1.95435 & 0.88069 & 28.06 & 2.71954 & 2.00744 \\
\hline
\end{tabular}

Table 7. Location, velocity and distance of orbit from both primaries for $q=1,0.995,0.99,0.9845$ for Sun-Mars system. 
Table 8. Location, velocity and distance of orbit from both primaries for $q=1,0.995,0.99,0.9845$ for Sun-Earth system.

\begin{tabular}{|c|c|c|c|c|c|c|c|c|c|c|c|c|c|c|c|c|}
\hline \multirow[t]{2}{*}{ No. of loop } & \multirow[t]{2}{*}{$\mathrm{C}$} & \multicolumn{2}{|c|}{$q=1$} & \multicolumn{5}{|c|}{$q=0.995$} & \multicolumn{4}{|c|}{$q=0.99$} & \multicolumn{4}{|c|}{$q=0.9845$} \\
\hline & & $\begin{array}{c}V \\
\left(\mathrm{~km} \cdot \mathrm{sec}^{-1}\right.\end{array}$ & $\begin{array}{c}D_{1} \\
\left(10^{7} \mathrm{~km}\right)\end{array}$ & $\begin{array}{c}D_{2} \\
\left(10^{8} \mathrm{~km}\right)\end{array}$ & $x$ & $\begin{array}{c}V \\
\left(\mathrm{~km} \cdot \mathrm{sec}^{-1}\right.\end{array}$ & $\begin{array}{c}D_{1} \\
\left(10^{7} \mathrm{~km}\right)\end{array}$ & $\begin{array}{c}D_{2} \\
\left(10^{8} \mathrm{~km}\right)\end{array}$ & $x$ & $\begin{array}{c}V \\
\left(\mathrm{~km} \cdot \mathrm{sec}^{-1}\right.\end{array}$ & $\begin{array}{c}D_{1} \\
\left(10^{7} \mathrm{~km}\right)\end{array}$ & $\begin{array}{c}D_{2} \\
\left(10^{8} \mathrm{~km}\right)\end{array}$ & $x$ & $\begin{array}{c}V \\
\left(\mathrm{~km} \cdot \mathrm{sec}^{-1}\right.\end{array}$ & $\begin{array}{c}D_{1} \\
\left(10^{7} \mathrm{~km}\right)\end{array}$ & $\begin{array}{c}D_{2} \\
\left(10^{8} \mathrm{~km}\right)\end{array}$ \\
\hline \multirow{4}{*}{1} & 2.960 .98305 & 35.32 & 0.25352 & 1.47064 & - & - & - & - & - & - & - & - & - & - & - & - \\
\hline & 2.950 .96795 & 35.70 & 0.47942 & 1.44805 & 0.9812 & 35.26 & 0.28120 & 1.46787 & 0.9949603 & 34.88 & 0.07535 & 1.48846 & - & - & - & - \\
\hline & 2.940 .9533 & 36.09 & 0.69858 & 1.42614 & 0.9661 & 35.64 & 0.50709 & 1.44529 & 0.9794 & 35.20 & 0.30813 & 1.46518 & 0.994601 & 34.77 & 0.08072 & 1.48792 \\
\hline & 2.930 .93904 & 36.47 & 0.91191 & 1.40480 & 0.95142 & 36.03 & 0.72671 & 1.42332 & 0.96425 & 35.59 & 0.53477 & 1.44252 & 0.97895 & 35.10 & 0.31486 & 1.46451 \\
\hline \multirow{4}{*}{2} & 2.960 .94157 & 34.74 & 0.87406 & 1.40859 & 0.9599 & 34.13 & 0.59985 & 1.43601 & - & - & - & - & - & & & \\
\hline & 2.950 .92259 & 35.30 & 1.15800 & 1.38019 & 0.93975 & 34.69 & 0.90129 & 1.40587 & 0.95813 & 34.07 & 0.62633 & 1.43336 & 0.98 & 33.38 & 2.99155 & 1.46608 \\
\hline & 2.940 .90456 & 35.83 & 1.42773 & 1.35322 & 0.92075 & 35.24 & 1.18553 & 1.37744 & 0.93796 & 34.64 & 0.97803 & 1.39819 & 0.9583 & 33.96 & 0.62378 & 1.43362 \\
\hline & 2.930 .8874 & 36.36 & 1.68445 & 1.32755 & 0.90273 & 35.78 & 1.45511 & 1.35048 & 0.91895 & 35.19 & 1.21246 & 1.37475 & 0.938 & 34.53 & 0.92747 & 1.40325 \\
\hline \multirow{4}{*}{3} & 2.960 .91538 & 34.72 & 1.26587 & 1.36941 & 0.93635 & 33.99 & 0.95215 & 1.40078 & 0.95965 & 33.22 & 6.03591 & 1.43564 & - & - & - & - \\
\hline & 2.950 .89434 & 35.37 & 1.58062 & 1.33793 & 0.91359 & 34.67 & 1.29264 & 1.36673 & 0.93462 & 33.94 & 0.97803 & 1.39819 & 0.96045 & 33.08 & 5.91623 & 1.43683 \\
\hline & 2.940 .87469 & 35.99 & 1.87459 & 1.30854 & 0.89252 & 35.32 & 1.60785 & 1.33521 & 0.91181 & 34.62 & 1.31927 & 1.36407 & 0.93513 & 33.81 & 0.97041 & 1.39895 \\
\hline & 2.930 .85623 & 36.60 & 2.15075 & 1.28092 & 0.87287 & 35.95 & 1.90181 & 1.30581 & 0.89074 & 35.27 & 1.63448 & 1.33255 & 0.9121 & 34.50 & 1.31493 & 1.36450 \\
\hline \multirow{4}{*}{4} & 2.960 .89714 & 34.82 & 1.53874 & 1.34212 & 0.91965 & 34.02 & 1.20199 & 1.37580 & 0.9452 & 33.15 & 8.19763 & 1.41402 & 0.97844 & 32.11 & 3.22492 & 1.46375 \\
\hline & 2.950 .87499 & 35.53 & 1.87010 & 1.30898 & 0.89535 & 34.78 & 1.56551 & 1.33944 & 0.91793 & 33.97 & 1.22772 & 1.37322 & 0.9463 & 33.01 & 8.03307 & 1.41566 \\
\hline & 2.940 .85454 & 36.21 & 2.17603 & 1.27839 & 0.8732 & 35.49 & 1.89688 & 1.30631 & 0.89359 & 34.73 & 1.59184 & 1.33681 & 0.91865 & 33.83 & 1.21695 & 1.37430 \\
\hline & 2.930 .83547 & 36.86 & 2.46132 & 1.24986 & 0.85274 & 36.16 & 2.20296 & 1.27570 & 0.87142 & 35.44 & 1.92351 & 1.30364 & 0.89403 & 34.59 & 1.58526 & 1.33747 \\
\hline \multirow{4}{*}{5} & 2.960 .88373 & 34.95 & 1.73935 & 1.32206 & 0.90719 & 34.10 & 1.38839 & 1.35716 & 0.93415 & 33.17 & 9.85071 & 1.39749 & 0.9703 & 32.01 & 4.44267 & 1.45157 \\
\hline & 2.950 .86099 & 35.70 & 2.08089 & 1.28791 & 0.88195 & 34.71 & 1.41427 & 1.35457 & 0.90546 & 34.05 & 1.41427 & 1.35457 & 0.93547 & 33.01 & 9.65323 & 1.39946 \\
\hline & 2.940 .84012 & 36.41 & 2.39175 & 1.25682 & 0.8592 & 35.65 & 2.10632 & 1.28536 & 0.88022 & 34.85 & 1.79186 & 1.31681 & 0.9063 & 33.91 & 1.40170 & 1.35582 \\
\hline & 2.930 .82075 & 37.09 & 2.68153 & 1.22784 & 0.83833 & 36.36 & 2.41853 & 1.25414 & 0.85745 & 35.60 & 2.13250 & 1.28274 & 0.88075 & 34.72 & 1.78393 & 1.31760 \\
\hline
\end{tabular}

$q$ is opposite in nature. For given value of $q$ and $C$ as number of loops increases, $D_{1}$ increases and $D_{2}$ decreases.

From Table 7, it is observed that single loop orbit for $q=1$ is closest to Mars and this distance is $7.521 \times 10^{7} \mathrm{~km}$. which is obtained using $C=2.96$ for $q=0.995$. Similar notations are used in Table 6 also.

Here $D_{1}$ is the distance of secondary body from Earth. From Table 8, it is observed that single loop orbit for $q=0.99$ is closest to Earth and this distance is $7.535 \times 10^{5} \mathrm{~km}$. This orbit is obtained by taking $C=2.95$.

We have analyzed stability of periodic orbits from loop 1 to 5 for $q=1,0.995,0.99$ and 0.9845 . Since stability behavior is similar for all these orbits, in this paper stability analysis for two loop orbit corresponding to $q=0.9845$ is given. Figure 23 shows stability region for $q=0.9845$ for two loop orbits. The left and right tips of the island are plotted by red and green curves, respectively. From Figure 23 it is clear that size of stability region is very small in comparison to " $P$ " family orbit [9]. So, these periodic orbits can be used as a transfer orbits as they are not stable. So, secondary body required few amount of fuel than Hohmann transfer. Figure 24 shows amplitude for two loop orbit when $q=0.9845$. It can be observe that there are two separatrices at $C=2.93$ and 2.95 


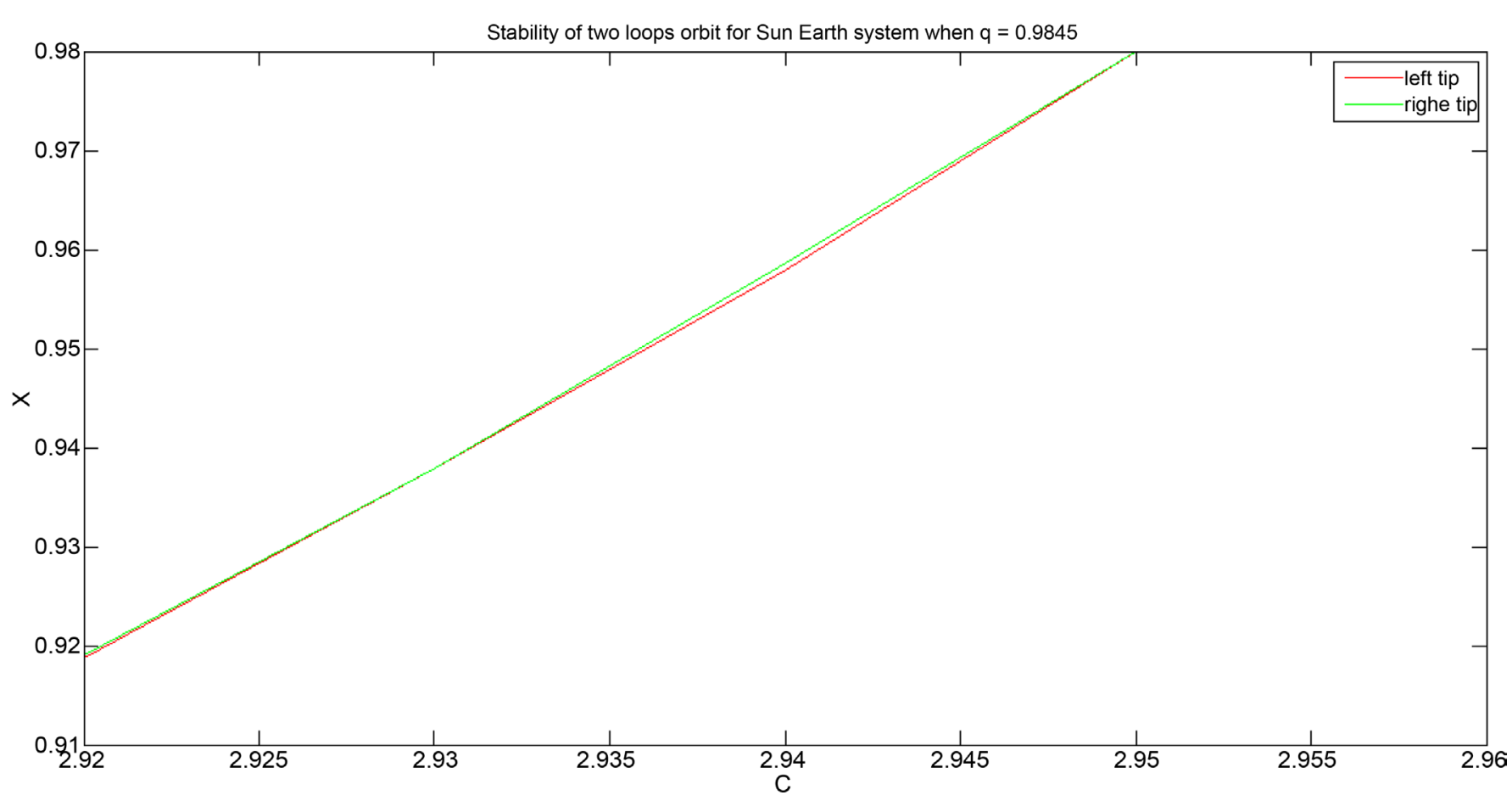

Figure 23. Stability analysis for two loops orbit for Sun-Earth system when $q=0.9845$.

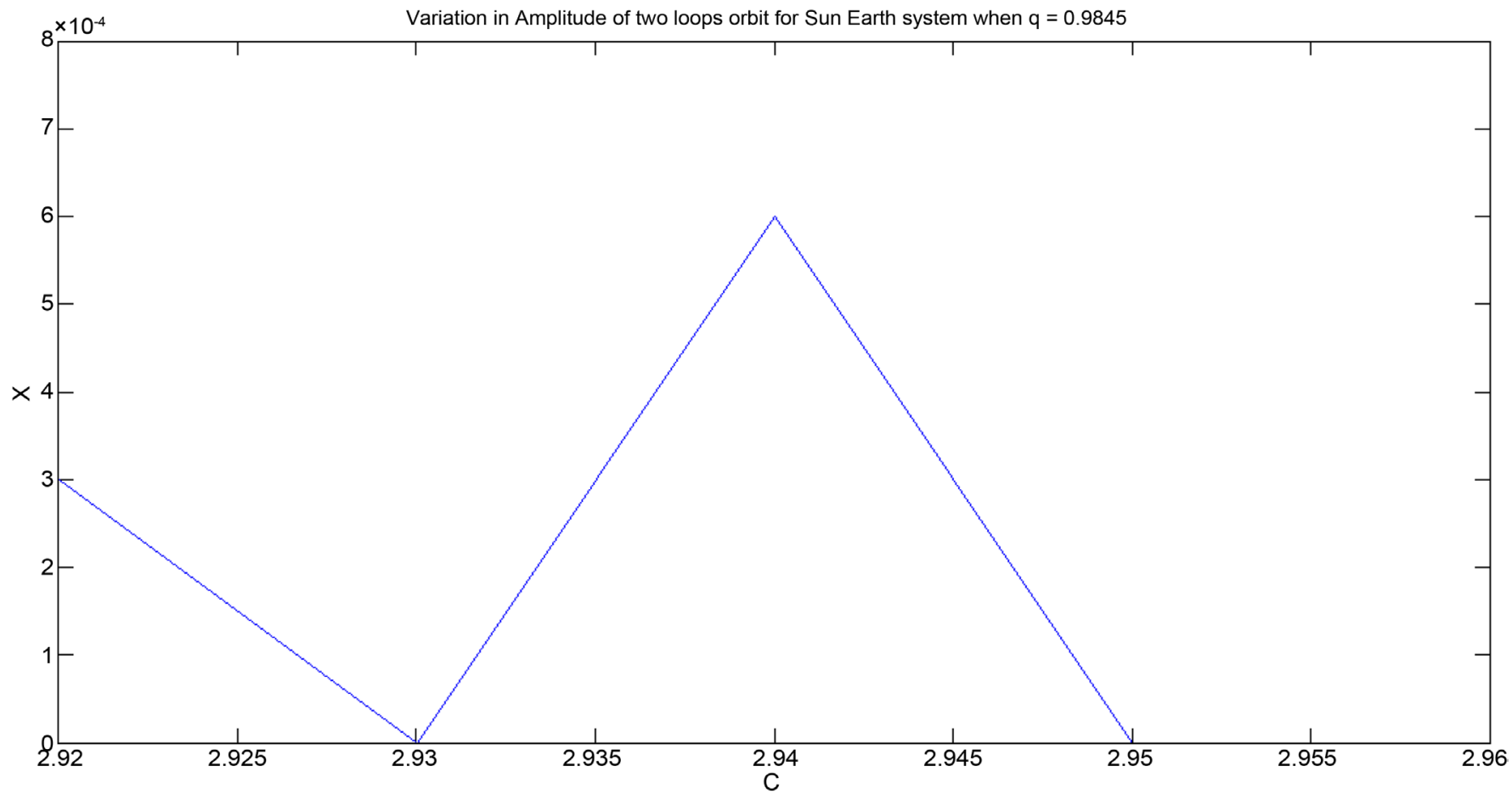

Figure 24. Amplitude for two loops orbit for Sun-Earth system when $q=0.9845$.

where stability of the periodic orbit is zero as the size of the island is zero. For $C=2.94$ we get maximum stability which is 0.0006 . Figure 25 shows size of the island for $C=$ 2.92 for two loop orbit when $q=0.9845$ which is 0.0003 where as Figure 26 shows PSS 


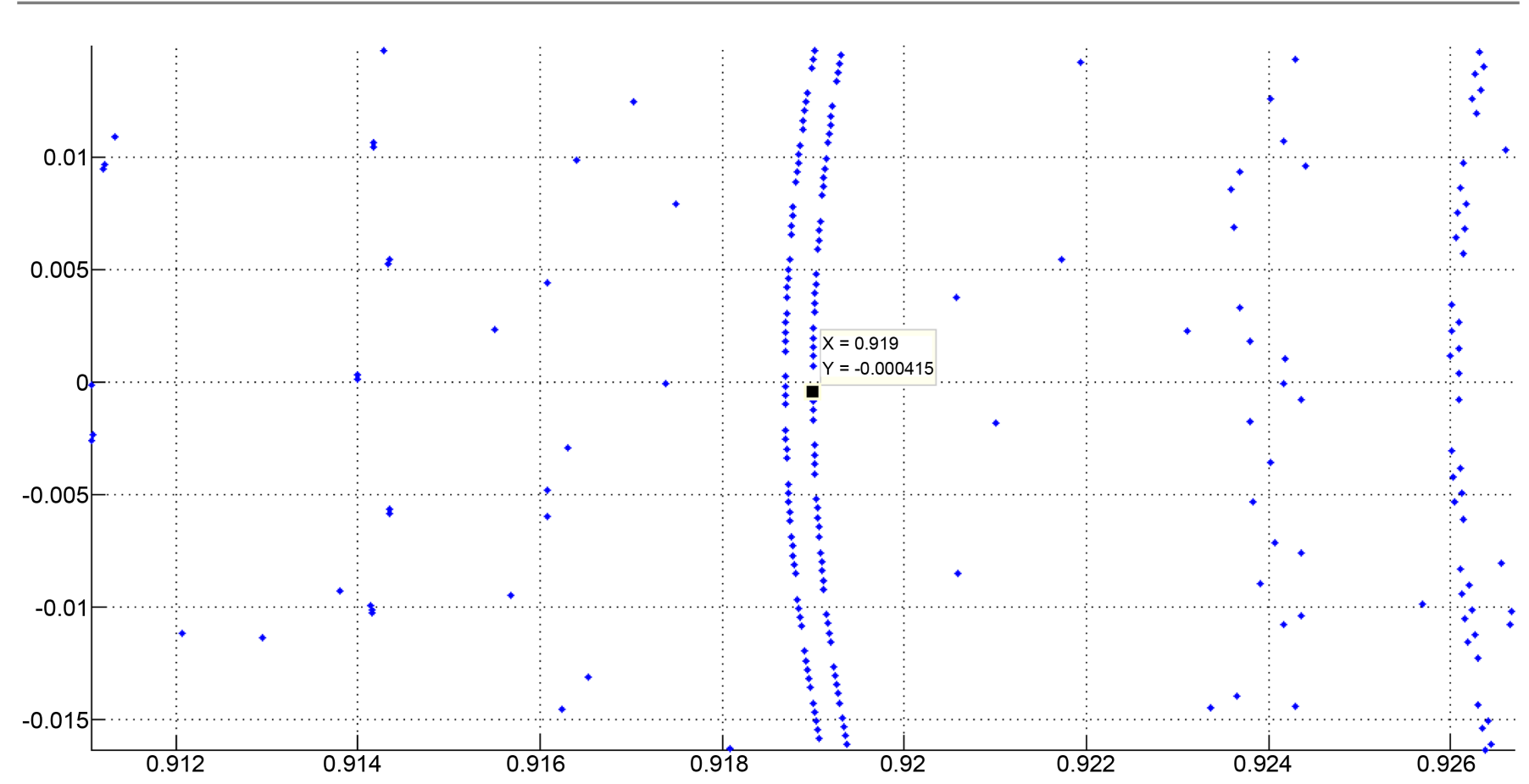

Figure 25. Enlarge view of PSS for $C=2.92$ when $q=0.9845$.

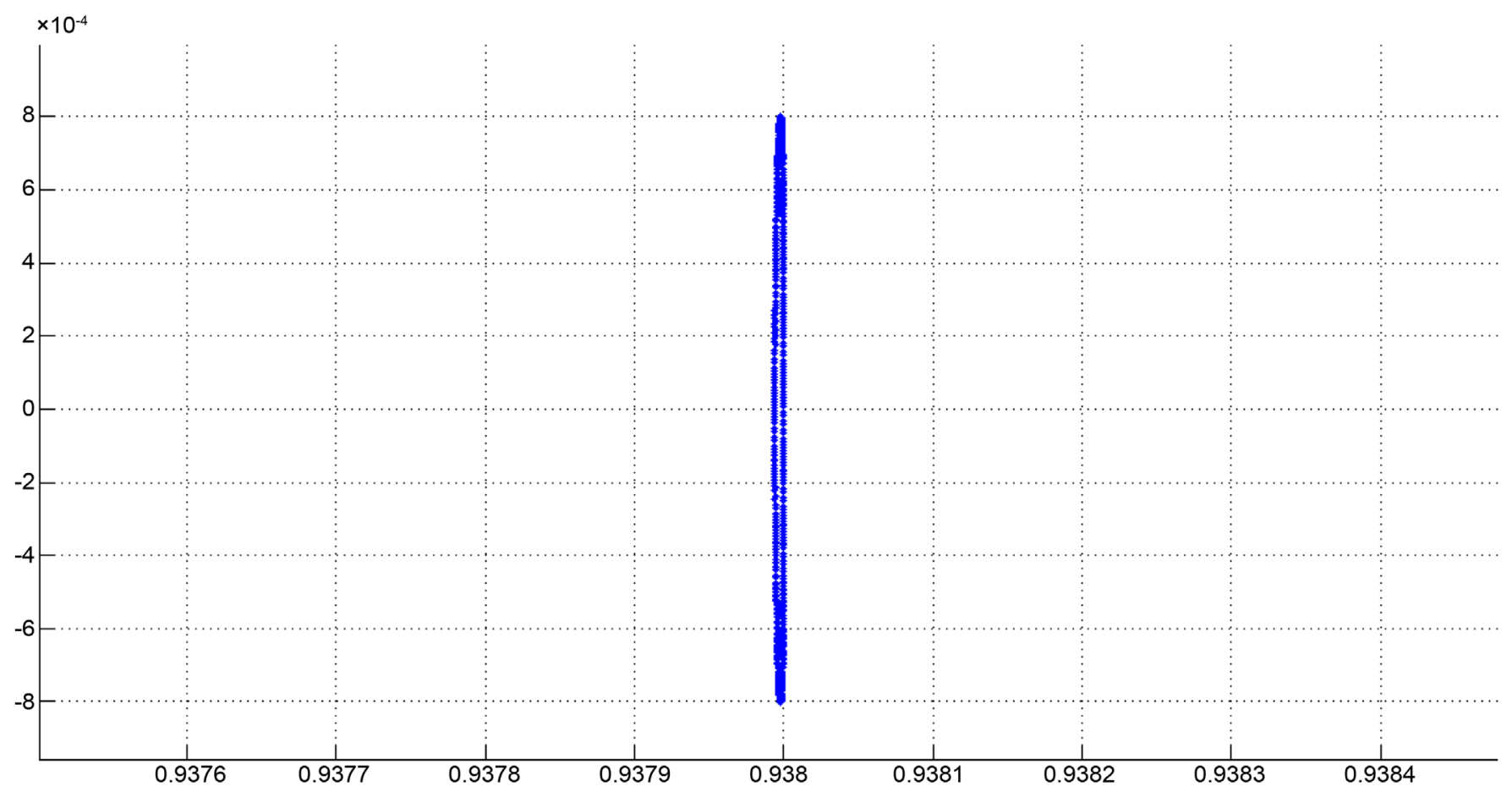

Figure 26. Enlarge view of PSS of first separatrix for two loop orbit for $C=2.93$ when $q=0.9845$.

of first separatrix at $C=2.93$ which is looks like a straight line where as for " $P$ " family orbit it is triangular due to third order resonance [9]. It can be seen that size of this island is zero. Figure 27 shows two loop orbit corresponding to first separatrix when $q=$ 0.9845. Figure 28 shows for $C=2.94$ again size of island increases and it becomes 


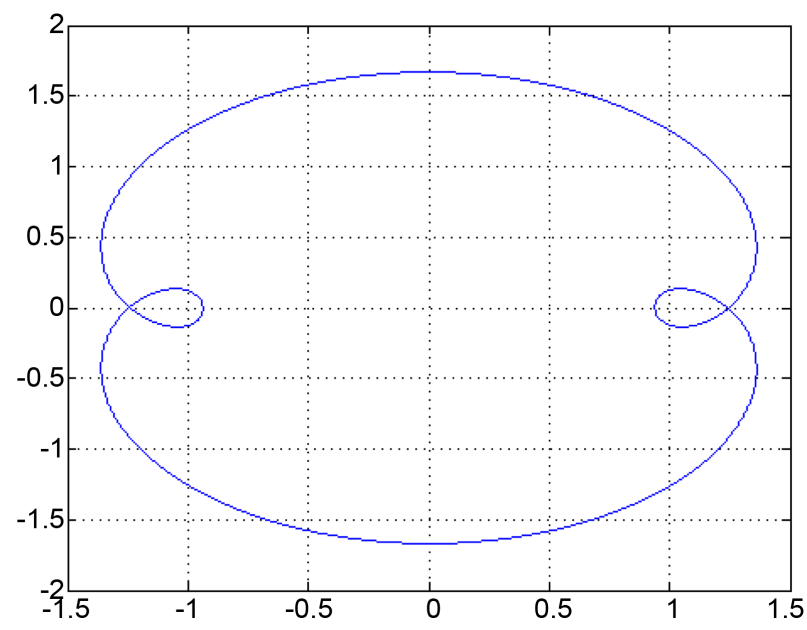

Figure 27. Orbit at first separatrix corresponding to $C=$ 2.93, $q=0.9845$ and $x=0.938$.

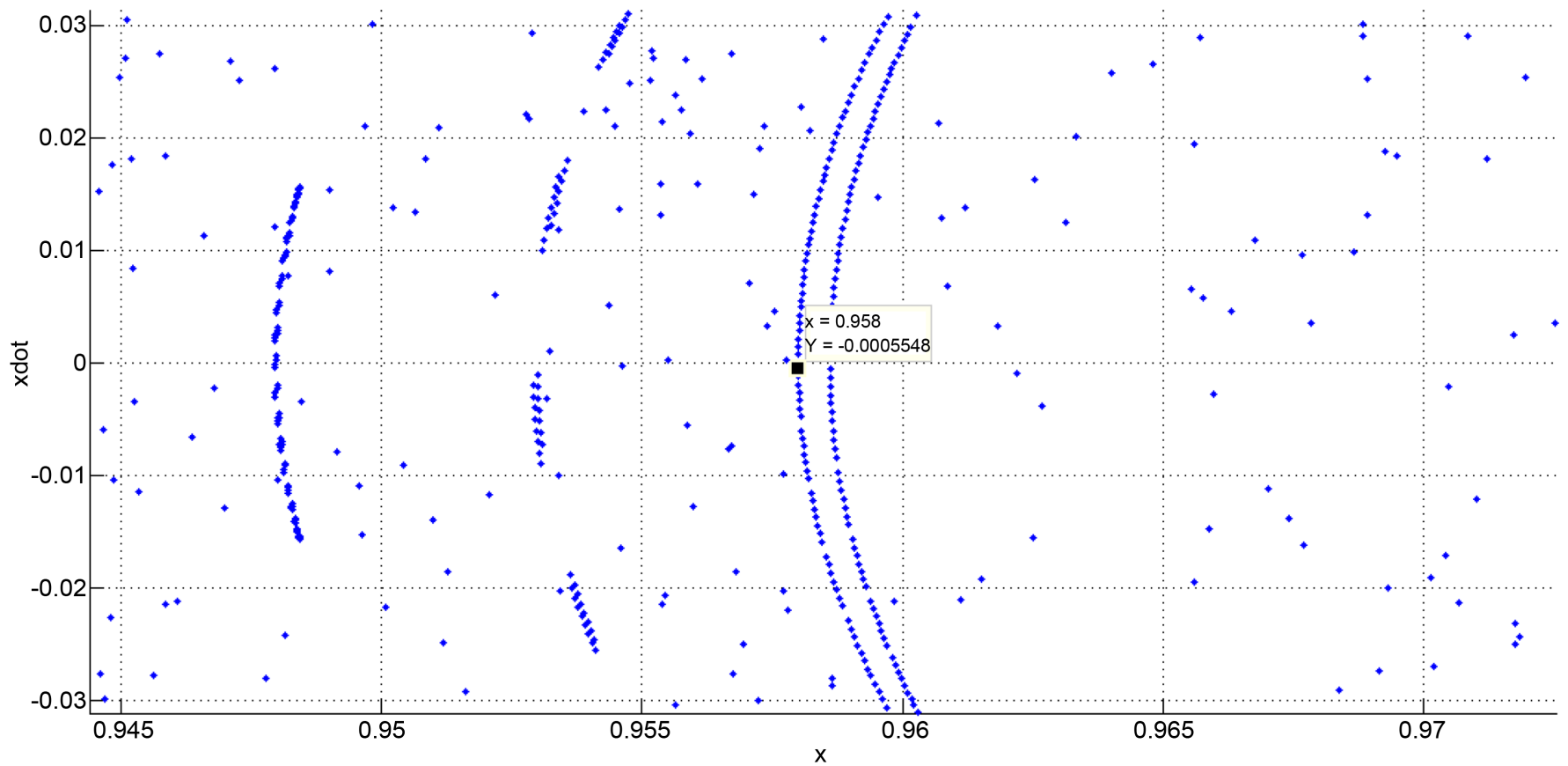

Figure 28. Enlarge view of PSS for $C=2.94$ when $q=0.9845$.

maximum, where as for $C=2.95$ again size of island becomes zero, which is second separatrix as shown in Figure 29. Two loop orbit corresponding to second separatrix is given in Figure 30.

\section{Conclusions}

We have investigated the effect of solar radiation pressure on the position, shape and size of closed periodic orbit with loops varying from 1 to 5 for Sun-Mars and Sun-Earth systems, respectively. A noticeable difference observed in both the systems is that for $C=$ 2.96, $q=0.995$, single-loop periodic orbit, for $C=2.96, q=0.99$, two loops orbit, for $C=$ 


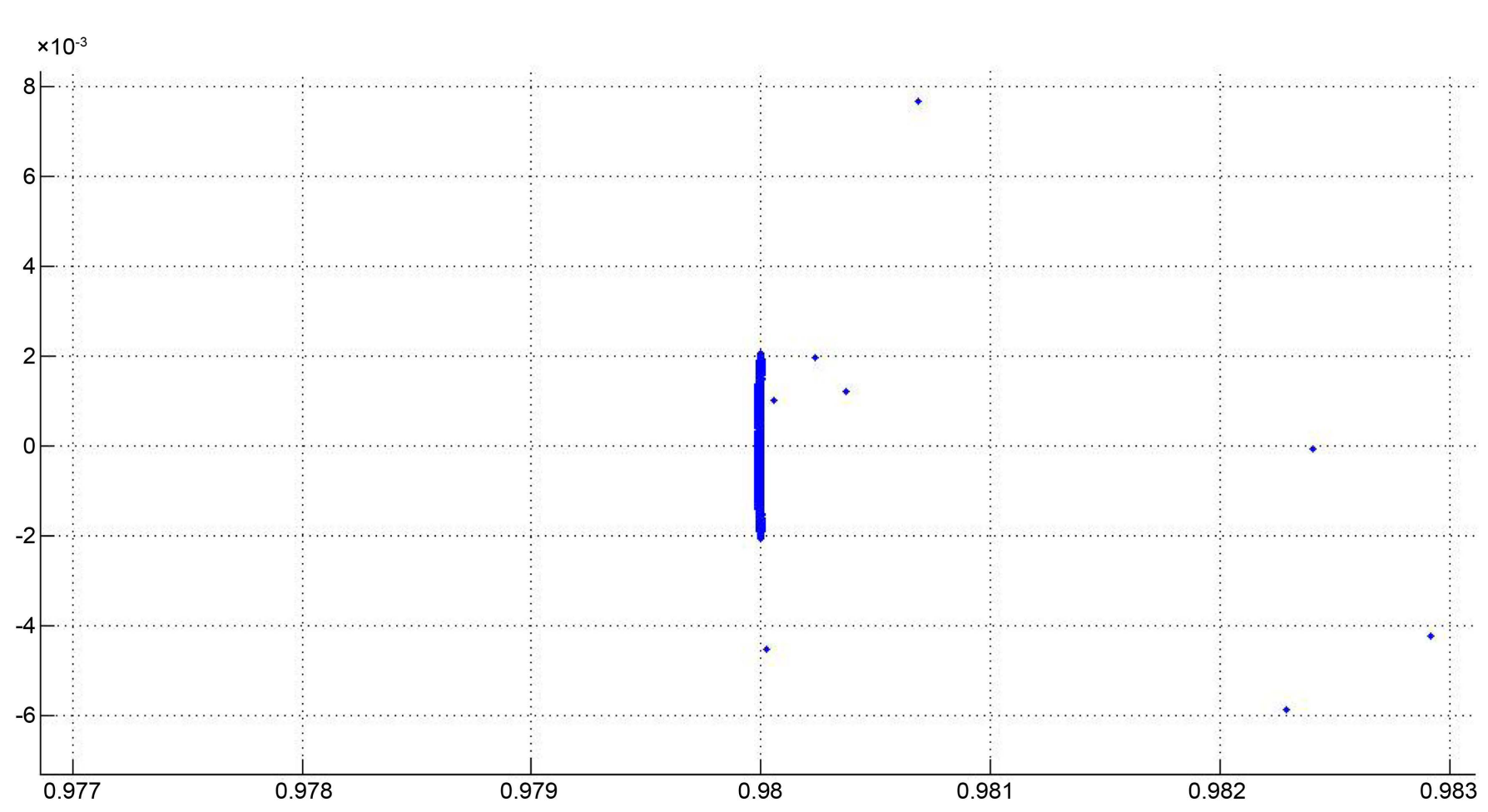

Figure 29. Enlarge view of PSS of second separatrix for two loop orbit for $C=2.95$ when $q=0.9845$.

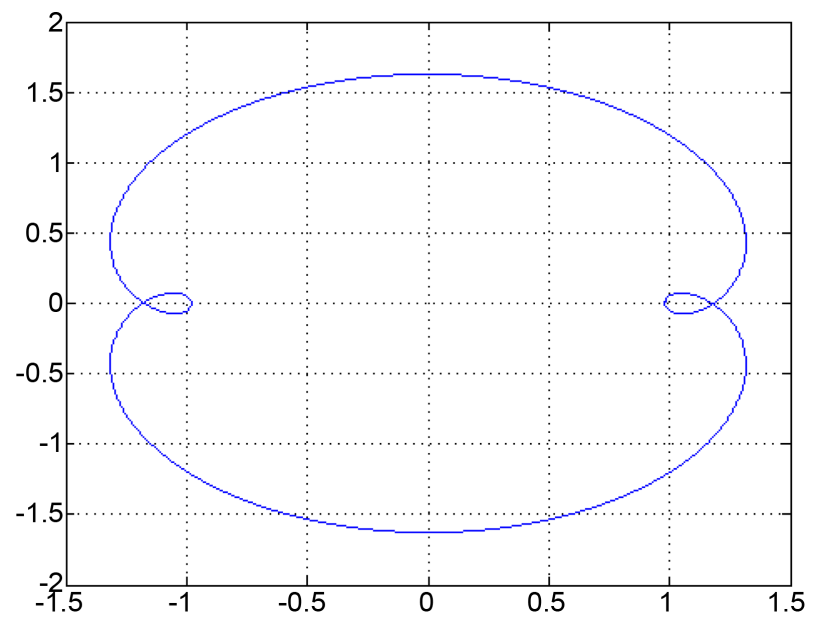

Figure 30. Orbit at second separatrix corresponding to $C=$ $2.95, q=0.9845$ and $x=0.98$.

2.96, $q=0.9845$, three loops periodic orbit does not exist for Sun-Earth system whereas it exists for Sun-Mars system.

The distance of closest approach of the infinitesimal particle from the smaller primary decreases with increase in solar radiation pressure from 1 to 0.9845 and distance between smaller primary and infinitesimal particle increases as number of loops increases for a given $C$ and $q$. It is found that the eccentricity decreases as number of loops increases. For a given number of loops, the eccentricity is found to decrease as solar radiation pressure increases from 1 to 0.9845 . Thus, the present analysis of the 
two systems-Sun-Mars and Sun-Earth systems-using PSS technique reveals that $q$ and $C$ has substantial effect on the position, shape and size of the obit.

It can be observed that for given solar radiation pressure and given number of loops, as Jacobi constant decreases, initial velocity of infinitesimal particle (space craft) and distance of infinitesimal particle (space craft) from second primary increase and distance of infinitesimal particle (space craft) from first primary body decreases. For given Jacobi constant and given number of loops, as solar radiation pressure increases from 1 to 0.9845 , initial velocity decreases and distance of infinitesimal particle (space craft) from second primary decreases. So, distance of infinitesimal particle (space craft) from first primary increases. Thus, the effect of Jacobi constant $C$ and solar radiation pressure $q$ is opposite in nature. For given value of solar radiation pressure $q$ and Jacobi constant, as number of loops increases, distance of infinitesimal particle (space craft) from second primary increases and distance of infinitesimal particle (space craft) from first primary decreases. It is further observed that for Sun-Mars system, single loop orbit for $q=0.995$ and $C=2.96$ is closest to Mars and this distance is $7.521 \times 10^{5} \mathrm{~km}$, whereas for Sun-Earth system, single loop orbit for $q=0.99$ and $C=2.95$ is closest to Earth and this distance is $7.535 \times 10^{5} \mathrm{~km}$. Since these orbits can be used for designing low-energy space mission. Hence detailed study is presented in this paper.

Stability analysis of this family of orbit indicates that these orbits having smaller stability region in comparison to " $f$ " family orbit. So, these orbits can be used as a transfer trajectory as less amount of fuel required for transferring of satellite from one orbit to another orbit. For each pair of $(q, C)$, there are two separatrices exist where stability of periodic orbit becomes zero.

\section{Acknowledgements}

The authors thank Mrs. Pooja Dutt, Applied Mathematics Division, Vikram Sarabhai Space Centre (ISRO), Thiruvananthapuram, India for her constructive comments.

\section{References}

[1] Koon, W.S., et al. (2001) Low Energy Transfer to the Moon. Celestial Mechanics and Dynamical Astronomy, 81, 63-73.

[2] Szebehely, V. (1967) Theory of Orbits. Academic Press, San Diego.

[3] Murray, C.D. and Dermot, S.F. (1999) Solar System Dynamics. Cambridge University Press, New York, USA.

[4] Dutt, P. and Sharma, R.K. (2010) Analysis of Periodic and Quasi-Periodic Orbits in the Earth-Moon System. Journal of Guidance, Control, and Dynamics, 33, 1010-1017.

[5] Dutt, P. and Sharma, R.K. (2011) Evolution of Periodic Orbits in the Sun-Mars System. Journal of Guidance, Control, and Dynamics, 34, 635-644.

[6] Safiyabeevi, A. and Sharma, R.K. (2011) Analysis of Periodic Orbits in the Saturn-Titan System Using the Method of Poincare Section Surfaces. Astrophysics and Space Science, 333, 37-48.

[7] Dutt, P. and Sharma, R.K. (2012) On the Evolution of the " $f$ " Family in the Restricted Three-Body Problem. Astrophysics and Space Science, 340, 63-70. 
[8] Pathak, N., Sharma, R.K. and Thomas, V.O. (2016) Evolution of Periodic Orbits in the Sun-Saturn System. International Journal of Astronomy and Astrophysics, 6, 175-197.

[9] Pathak, N. and Thomas, V.O. (2016) Evolution of the "f" Family Orbits in the Photo Gravitational Sun-Saturn System with Oblateness. International Journal of Astronomy and Astrophysics, 9, 254-271.

[10] Stromgren, E. (1935) O. Connaissance Actualle des Orbites dans le Problem des Trois Corps. Vol. 9, Publications and Minor Communications of Copenhagen Observatory, Publication 100, Copenhagen University, Astronomical Observatory, Denmark, 87.

[11] Sharma, R.K. (1987) The Linear Stability of Libration Points of the Photogravitational Restricted Three-Body Problem When the Smaller Primary Is an Oblate Spheroid. Astrophysics and Space Science, 135, 271-281.

[12] Koon, W.S., et al. (2011) Dynamical Systems, the Three-Body Problem and Space Mission Design. $\underline{\text { http://www.cds.caltech.edu/ marsden/books/Mission_Design.html }}$

Submit or recommend next manuscript to SCIRP and we will provide best service for you:

Accepting pre-submission inquiries through Email, Facebook, LinkedIn, Twitter, etc. A wide selection of journals (inclusive of 9 subjects, more than 200 journals)

Providing 24-hour high-quality service

User-friendly online submission system

Fair and swift peer-review system

Efficient typesetting and proofreading procedure

Display of the result of downloads and visits, as well as the number of cited articles Maximum dissemination of your research work

Submit your manuscript at: http://papersubmission.scirp.org/

Or contact ijaa@scirp.org 